Supporting Information for

\title{
A General Method for Synthesizing Pyranoid Glycals. A New Route to Allal and Gulal Derivatives
}

Omar Boutureira, Miguel Angel Rodríguez, M. Isabel Matheu, Yolanda Díaz*, Sergio Castillón*

Departament de Química Anlítica i Química Orgànica, Facultat de Química, C/ Marcel.lí Domingo s/n, 43007 Tarragona, Spain. 


\section{Table of contents}

1. General Procedure for synthesizing glycals 3

2. Synthesis of 6

3. Synthesis of $\mathbf{1 4}$

4. Synthesis of $\mathbf{1 5} 4$

5. Synthesis of $\mathbf{1 7} 5$

6. Synthesis of $\mathbf{1 8} 6$

7. Synthesis of 19

8. Synthesis of 22

9. ${ }^{1} \mathrm{H}$ NMR of 5

10. ${ }^{13} \mathrm{C}$ NMR of 5

11. ${ }^{1} \mathrm{H}$ NMR of $6 \quad 10$

12. ${ }^{13} \mathrm{C}$ NMR of 6

13. ${ }^{1} \mathrm{H}$ NMR of 7

14. ${ }^{13} \mathrm{C}$ NMR of 7

15. ${ }^{1} \mathrm{H}$ NMR of $\mathbf{8}$

16. ${ }^{13} \mathrm{C}$ NMR of 8

17. ${ }^{1} \mathrm{H}$ NMR of $\mathbf{1 0} 16$

18. ${ }^{13} \mathrm{C}$ NMR of $\mathbf{1 0} \quad 17$

19. ${ }^{1} \mathrm{H}$ NMR of $\mathbf{1 1} 18$

20. ${ }^{1} \mathrm{H}$ NMR of $\mathbf{1 2} 19$

21. ${ }^{1} \mathrm{H}$ NMR of $\mathbf{1 4} 20$

22. ${ }^{13} \mathrm{C}$ NMR of $\mathbf{1 4}$

23. ${ }^{1} \mathrm{H}$ NMR of $\mathbf{1 5} 22$

24. ${ }^{13} \mathrm{C}$ NMR of $\mathbf{1 5} 23$

25. ${ }^{1} \mathrm{H}$ NMR of $\mathbf{1 7}$

26. ${ }^{13} \mathrm{C}$ NMR of $\mathbf{1 7} 25$

27. ${ }^{1} \mathrm{H}$ NMR of $\mathbf{1 8} 26$

38. ${ }^{13} \mathrm{C}$ NMR of $\mathbf{1 8} 27$

39. ${ }^{1} \mathrm{H}$ NMR of 19

30. ${ }^{13} \mathrm{C}$ NMR of $\mathbf{1 9} 29$

31. References 30 


\section{General Procedure for synthesizing glycals from 2-deoxy-2-iodo-1-thio-glycosides}

The 2-deoxy-2-iodo-pyranoside derivative $(1 \mathrm{mmol})$ and $\mathrm{NaOAc}(1.4 \mathrm{mmol})$ were dissolved in a mixture of THF $(1.7 \mathrm{~mL})$ and acetic acid $(63 \mu \mathrm{L})$ and cooled to 0 ${ }^{\circ} \mathrm{C} . \mathrm{Zn} / \mathrm{Cu}$ couple $(661 \mathrm{mg})$ was then added and the reaction was allowed to reach room temperature. The mixture was then diluted with dichloromethane and washed with a saturated solution of $\mathrm{NaHCO}_{3}$. The combined aqueous layer was extracted with dichloromethane, and the combination of organic layers was dried with $\mathrm{MgSO}_{4}$ and concentrated. The residue was purified by chromatographic techniques.

\section{3,4,6-tri- $O$-benzyl-D-allal (3,4,6-tri- $O$-benzyl-D-ribo-hex-1-enitol) $(6){ }^{1}$}

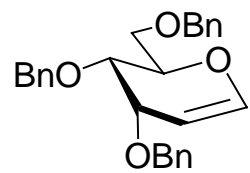

The title compound was prepared following the general procedure above starting from phenyl 3,4,6-tri-O-benzyl-2-deoxy-2-iodo-1-thio- $\alpha$-D-allo-pyranoside (5) ( $\alpha: \beta$ 1:9) (75 $\mathrm{mg}, 0.11 \mathrm{mmol})$ in a mixture of $0.2 \mathrm{~mL}$ THF, $10 \mu \mathrm{L}$ acetic acid, NaOAc (13 mg, 0.16 $\mathrm{mmol}$ ) and $72.7 \mathrm{mg} \mathrm{Zn/Cu}$ couple. The reaction was monitored by TLC (EtOAc/hexane 1:3) and was allowed to reach room temperature over a period of $6 \mathrm{~h}$. The crude product was purified by radial chromatography (from hexane to EtOAc/hexane 1:3) to afford the product 6 (48 $\mathrm{mg}, 100 \%)$ as a yellowish syrup.

$\mathrm{R}_{\mathrm{f}}($ EtOAc/hexane 1:3): 0.43.

Anal. Calcd for $\mathrm{C}_{27} \mathrm{H}_{28} \mathrm{O}_{4}: 77.86 \% \mathrm{C}, 6.78 \% \mathrm{H}$. Found: $77.70 \% \mathrm{C}, 6.70 \% \mathrm{H}$. $\mathrm{RMN}{ }^{1} \mathrm{H}\left(\mathrm{CDCl}_{3}, 400 \mathrm{MHz}\right) \delta$ in ppm: 7.36-7.25 $(15 \mathrm{H}, \mathrm{m}, \mathrm{Ar}), 6.47\left(1 \mathrm{H}, \mathrm{d}, \mathrm{J}_{1,2}=6 \mathrm{~Hz}\right.$, $\mathrm{H}-1), 4.90\left(1 \mathrm{H}, \mathrm{dd}, \mathrm{J}_{1,2}=\mathrm{J}_{2,3}=6 \mathrm{~Hz}, \mathrm{H}-2\right), 4.72\left(1 \mathrm{H}, \mathrm{d}, \mathrm{J}_{\mathrm{AB}}=12 \mathrm{~Hz}, \mathrm{CH}_{2} \mathrm{Ph}\right), 4.64(1 \mathrm{H}, \mathrm{d}$, $\left.\mathrm{J}_{\mathrm{AB}}=11.6 \mathrm{~Hz}, \mathrm{CH}_{2} \mathrm{Ph}\right), 4.63\left(1 \mathrm{H}, \mathrm{d}, \mathrm{J}_{\mathrm{AB}}=12 \mathrm{~Hz}, \mathrm{CH}_{2} \mathrm{Ph}\right), 4.60\left(1 \mathrm{H}, \mathrm{d}, \mathrm{J}_{\mathrm{AB}}=11.6 \mathrm{~Hz}\right.$, $\left.\mathrm{CH}_{2} \mathrm{Ph}\right), 4.59\left(1 \mathrm{H}, \mathrm{d}, \mathrm{J}_{\mathrm{AB}}=11.6 \mathrm{~Hz}, \mathrm{CH}_{2} \mathrm{Ph}\right), 4.47\left(1 \mathrm{H}, \mathrm{d}, \mathrm{J}_{\mathrm{AB}}=11.6 \mathrm{~Hz}, \mathrm{CH}_{2} \mathrm{Ph}\right), 4.31$ $\left(1 \mathrm{H}\right.$, app. $\mathrm{t}$ (full paper), $\left.\mathrm{J}_{4,5}=10.4 \mathrm{~Hz}, \mathrm{H}-5\right), 3.96\left(1 \mathrm{H}, \mathrm{dd}, \mathrm{J}_{2,3}=5.2 \mathrm{~Hz}, \mathrm{~J}_{3,4}=3.6 \mathrm{~Hz}, \mathrm{H}-\right.$ 3), 3.85 (2H, m, H-6a,b), 3.79 (1H, dd, J $\left.{ }_{4,5}=10.4 \mathrm{~Hz}, \mathrm{~J}_{3,4}=3.6 \mathrm{~Hz}, \mathrm{H}-4\right)$. $\mathrm{RMN}{ }^{13} \mathrm{C}\left(\mathrm{CDCl}_{3}, 100.6 \mathrm{MHz}\right) \delta$ in ppm: 146.9 (C-1), 138.8-138.1 (C, Ar), 128.6-127.8 (CH, Ar), 98.3 (C-2), $74.0(\mathrm{C}-4), 73.8\left(\mathrm{CH}_{2} \mathrm{Ph}\right), 73.2(\mathrm{C}-5), 71.5,70.5\left(2 \mathrm{CH}_{2} \mathrm{Ph}\right), 69.0$ (C-6), $65.5(\mathrm{C}-3)$. 


\section{3,4,6-tri- $O$-benzyl-D-gulal (3,4,6-tri- $O$-benzyl-D-xylo-hex-1-enitol) (14).}

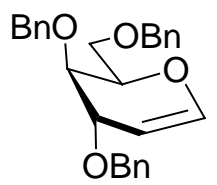

The title compound was prepared following the general procedure starting from the phenyl 3,4,6-tri-O-benzyl-2-deoxy-2-iodo-1-thio- $\alpha$-D-gulo-pyranoside (7) ( $\alpha: \beta$ 10:1) (159 mg, $0.24 \mathrm{mmol})$ in a mixture of $0.4 \mathrm{~mL}$ THF, $20 \mu \mathrm{L}$ acetic acid, NaOAc (28 $\mathrm{mg}, 0.34 \mathrm{mmol}$ ) and $161 \mathrm{mg} \mathrm{Zn/Cu}$ couple for $1 \mathrm{~h}$. The reaction was monitored by TLC (EtOAc/hexane 1:3). The crude product was purified by radial chromatography (EtOAc/hexane 1:4) to afford the product $\mathbf{1 4}(50 \mathrm{mg}, 92 \%)$ as a colorless syrup.

$\mathrm{R}_{\mathrm{f}}($ EtOAc/hexane 1:3): 0.46.

$[\alpha]_{\mathrm{D}}^{25}=64.3\left(\mathrm{CH}_{2} \mathrm{Cl}_{2}, c=2\right)$

Anal. Calcd for $\mathrm{C}_{27} \mathrm{H}_{28} \mathrm{O}_{4}: 77.86 \% \mathrm{C}, 6.78 \% \mathrm{H}$. Found: $77.80 \% \mathrm{C}, 6.74 \% \mathrm{H}$

$\mathrm{RMN}{ }^{1} \mathrm{H}\left(\mathrm{CDCl}_{3}, 400 \mathrm{MHz}\right) \delta$ in ppm: 7.36-7.21 $(10 \mathrm{H}, \mathrm{m}, \mathrm{Ar}), 6.36\left(1 \mathrm{H}, \mathrm{ddd}, \mathrm{J}_{1,2}=6.4\right.$ $\left.\mathrm{Hz}, \mathrm{J}_{1,3 \mathrm{a}}=1.6 \mathrm{~Hz}, \mathrm{~J}_{1,3 \mathrm{~b}}=2.4 \mathrm{~Hz}, \mathrm{H}-1\right), 4.63\left(1 \mathrm{H}, \mathrm{ddd}, \mathrm{J}_{2,1}=6.4 \mathrm{~Hz} ., \mathrm{J}_{2,3 \mathrm{a}}=5.2 \mathrm{~Hz} ., \mathrm{J}_{2,3 \mathrm{~b}}=\right.$ $2.6 \mathrm{~Hz} ., \mathrm{H}-2), 4.62-4.50\left(4 \mathrm{H}, \mathrm{m}, \mathrm{J}_{\mathrm{AB}}=9.0 \mathrm{~Hz} ., \mathrm{J}_{\mathrm{AB}}\right.$ = $\left.12.0 \mathrm{~Hz} ., 4 \mathrm{CH}_{2} \mathrm{Ph}\right), 3.90(1 \mathrm{H}$, ddd, $\left.\mathrm{J}_{5,4}=8.0 \mathrm{~Hz} ., \mathrm{J}_{5,6 \mathrm{a}}=\mathrm{J}_{5,6 \mathrm{~b}}=4.0 \mathrm{~Hz} ., \mathrm{H}-5\right), 3.79$ (1H, m, H-4, H-6a, H-6b), 2.38 (1H, dddd, $\left.\mathbf{J}_{3 \mathrm{a}, 3 \mathrm{~b}}=16.4 \mathrm{~Hz} ., \mathbf{J}_{3 \mathrm{a}, 2}=5.2 \mathrm{~Hz} ., \mathrm{J}_{3 \mathrm{a}, 4}=6.0 \mathrm{~Hz} ., \mathrm{J}_{3 \mathrm{a}, 1}=1.6 \mathrm{~Hz} ., \mathrm{H}-3 \mathrm{a}\right), 2.08$ (1H, dddd, $\left.\mathrm{J}_{3 \mathrm{~b}, 3 \mathrm{a}}=16.4 \mathrm{~Hz} ., \mathrm{J}_{3 \mathrm{~b}, 2}=2.6 \mathrm{~Hz} ., \mathrm{J}_{3 \mathrm{~b}, 4}=8.4 \mathrm{~Hz} ., \mathrm{J}_{3 \mathrm{~b}, 1}=2.4 \mathrm{~Hz} ., \mathrm{H}-3 \mathrm{~b}\right)$.

$\mathrm{RMN}{ }^{13} \mathrm{C}\left(\mathrm{CDCl}_{3}, 100.6 \mathrm{MHz}\right) \delta$ in ppm: $143.3(\mathrm{C}-1), 138.4-127.8\left(\mathrm{C}_{\text {aromatic }}\right), 97.8(\mathrm{C}-$ 2), 76.9 (C-5), 73.7, 71,3 (2 $\left.\mathrm{CH}_{2} \mathrm{Ph}\right), 70.7$ (C-4), 69.2 (C-6), 26.7 (C-3).

\section{6-O-tert-butyldiphenylsilyl-3,4-O-isopropylidene-D-galactal} (6-O-tert-butyldiphenylsilyl-3,4-O-isopropylidene-D-lyxo-hex-1-enitol) (15). ${ }^{2}$

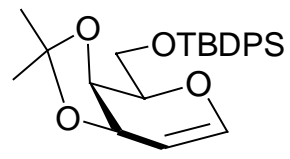

The title compound was prepared following the general procedure above starting from 8 ( $\alpha: \beta 40: 1)(200 \mathrm{mg}, 0.30 \mathrm{mmol})$ in a mixture of $0.5 \mathrm{~mL}$ THF, $24 \mu \mathrm{L}$ acetic acid, $\mathrm{NaOAc}(36 \mathrm{mg}, 0.42 \mathrm{mmol}$ ) and $200 \mathrm{mg} \mathrm{Zn} / \mathrm{Cu}$ couple for $1 \mathrm{~h}$. The reaction was monitored by TLC $\left(\mathrm{CH}_{2} \mathrm{Cl}_{2}\right)$. The crude product was purified by radial chromatography (from hexane to EtOAc/hexane 1:3) to afford the expected product $15(110 \mathrm{mg}, 86 \%)$ as a colorless syrup. 
$\mathrm{R}_{\mathrm{f}}\left(\mathrm{CH}_{2} \mathrm{Cl}_{2}\right): 0.55$.

Anal. Calcd for $\mathrm{C}_{25} \mathrm{H}_{32} \mathrm{O}_{4} \mathrm{Si}: 70.75 \% \mathrm{C}, 7.55 \% \mathrm{H}$. Found: $70.89 \% \mathrm{C}, 7.70 \% \mathrm{H}$

$\mathrm{RMN}^{1} \mathrm{H}\left(\mathrm{CDCl}_{3}, 400 \mathrm{MHz}\right) \delta$ in ppm: 7.70-7.38 $(10 \mathrm{H}, \mathrm{m}, \mathrm{Ar}), 6.34\left(1 \mathrm{H}, \mathrm{d}, \mathrm{J}_{1,2}=6 \mathrm{~Hz}\right.$, H-1), $4.76(1 \mathrm{H}, \mathrm{m}, \mathrm{H}-2), 4.66\left(1 \mathrm{H}, \mathrm{dd}, \mathrm{J}_{3,4}=6 \mathrm{~Hz}, \mathrm{~J}_{2,3}=2.4 \mathrm{~Hz}, \mathrm{H}-3\right), 4.56\left(1 \mathrm{H}, \mathrm{d}, \mathrm{J}_{3,4}=6\right.$ Hz, H-4), $4.02\left(1 \mathrm{H}, \mathrm{dd}, \mathrm{J}_{5,6 \mathrm{a}}=14 \mathrm{~Hz}, \mathrm{~J}_{5,6 \mathrm{~b}}=7.2 \mathrm{~Hz}, \mathrm{H}-5\right), 3.99-3.91$ (2H, m, H-6a,b), 1.45, 1.37, (6H, s, $2 \mathrm{CH}_{3}$, isoprop.), $1.07\left(9 \mathrm{H}, \mathrm{s}, 3 \mathrm{CH}_{3}, t-\mathrm{Bu}\right)$.

$\mathrm{RMN}{ }^{13} \mathrm{C}\left(\mathrm{CDCl}_{3}, 100.6 \mathrm{MHz}\right) \delta$ in ppm: $144.8(\mathrm{C}-1), 135.8-127.9(\mathrm{C}, \mathrm{CH}, \mathrm{Ar}), 110.4$ $\left(\mathrm{C}_{\text {ketal }}\right), 102.8$ (C-2), 75.1 (C-5), 72.0 (C-4), 68.7 (C-3), 63.1 (C-6), 28.4, $27.0\left(2 \mathrm{CH}_{3}\right.$, isoprop.), $27.0\left(3 \mathrm{CH}_{3}, t-\mathrm{Bu}\right), 19.5(\mathrm{C}, t-\mathrm{Bu})$.

3,4:6,7-di- $O$-isopropylidene-D-glycero-D-talal (17). ${ }^{3}$

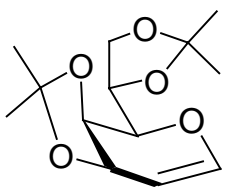

The title compound was prepared following the general procedure above starting from $10(\alpha: \beta$ 1:0) (105 mg, $0.21 \mathrm{mmol})$ in a mixture of $0.4 \mathrm{~mL}$ THF, $18 \mu \mathrm{L}$ acetic acid, $\mathrm{NaOAc}(24.5 \mathrm{mg}, 0.30 \mathrm{mmol})$ and $141 \mathrm{mg} \mathrm{Zn} / \mathrm{Cu}$ couple for $1 \mathrm{~h}$. The reaction was monitored by TLC (EtOAc/hexane 1:3). The crude product was purified by radial chromatography (EtOAc/hexane 1:4) to afford the expected product 17 (76.6 mg, 97\%) as a white foam.

$\mathrm{R}_{\mathrm{f}}($ EtOAc/hexane 1:3): 0.28.

Anal. Calcd for $\mathrm{C}_{13} \mathrm{H}_{20} \mathrm{O}_{5}: 60.92 \% \mathrm{C}, 7.87 \% \mathrm{H}$. Found: $61.01 \% \mathrm{C}, 7.90 \% \mathrm{H}$

$\mathrm{RMN}{ }^{1} \mathrm{H}\left(\mathrm{CDCl}_{3}, 400 \mathrm{MHz}\right) \delta$ in ppm: $6.36\left(1 \mathrm{H}, \mathrm{d}, \mathrm{J}_{1,2}=6.4 \mathrm{~Hz}, \mathrm{H}-1\right), 4.80(1 \mathrm{H}, \mathrm{ddd}$, $\left.\mathbf{J}_{1,2}=6.4 \mathrm{~Hz}, \mathbf{J}_{2,3}=2.8 \mathrm{~Hz}, \mathbf{J}_{2,4}=1.0 \mathrm{~Hz}, \mathrm{H}-2\right), 4.67\left(1 \mathrm{H}, \mathrm{dd}, \mathbf{J}_{3,4}=6.0 \mathrm{~Hz}, \mathrm{~J}_{2,3}=2.8 \mathrm{~Hz}, \mathrm{H}-\right.$ 3), $4.43\left(1 \mathrm{H}, \mathrm{dd}, \mathbf{J}_{3,4}=6.0 \mathrm{~Hz}, \mathrm{~J}_{2,4}=1.0 \mathrm{~Hz}, \mathrm{H}-4\right), 4.38(1 \mathrm{H}, \mathrm{m}, \mathrm{H}-6), 4.12\left(1 \mathrm{H}, \mathrm{dd}, \mathrm{J}_{7 \mathrm{a}, \mathrm{b}}=\right.$ $\left.8.4 \mathrm{~Hz}, \mathrm{~J}_{6,7 \mathrm{a}}=6.0 \mathrm{~Hz}, \mathrm{H}-7 \mathrm{a}\right), 4.08\left(1 \mathrm{H}, \mathrm{dd}, \mathrm{J}_{7 \mathrm{a}, \mathrm{b}}=8.4 \mathrm{~Hz}, \mathrm{~J}_{7 \mathrm{a}, 6}=5.0 \mathrm{~Hz}, \mathrm{H}-7 \mathrm{a}\right), 3.78(1 \mathrm{H}$, $\left.\mathrm{d}, \mathrm{J}_{5,6}=8.0 \mathrm{~Hz}, \mathrm{H}-5\right), 1.46,1.44,1.39\left(12 \mathrm{H}, \mathrm{s}, 4 \mathrm{CH}_{3}\right)$.

$\mathrm{RMN}{ }^{13} \mathrm{C}\left(\mathrm{CDCl}_{3}, 100.6 \mathrm{MHz}\right) \delta$ in ppm: 144.7 (C-1), 110.8, $109.6\left(2 \mathrm{C}_{\text {ketal }}\right), 103.2(\mathrm{C}$ 2), 75.2 (C-3), 74.3 (C-4), 72.2 (C-6), 68.6 (C-5), 66.9 (C-7), 28.3, 27.3, 27.1, 25.5 $\left(4 \mathrm{CH}_{3}\right)$. 


\section{3,4-O-isopropylidene-D-arabinal (3,4-O -isopropylidene-D-erythro-pent-1-enitol)}

(18). ${ }^{4}$

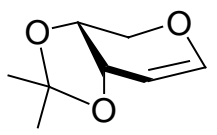

The title compound was prepared following the general procedure above starting from $11(\alpha: \beta 12: 1)(55 \mathrm{mg}, 0.14 \mathrm{mmol})$ in a mixture of $240 \mu \mathrm{L}$ THF, $11 \mu \mathrm{L}$ acetic acid, $\mathrm{NaOAc}(16 \mathrm{mg}, 0.20 \mathrm{mmol})$ and $93 \mathrm{mg} \mathrm{Zn} / \mathrm{Cu}$ couple for $1.5 \mathrm{~h}$. The reaction was monitored by TLC $\left(\mathrm{CH}_{2} \mathrm{Cl}_{2}\right)$. The crude product was purified by flash chromatography $\left(\mathrm{CH}_{2} \mathrm{Cl}_{2}\right)$ to afford the product $\mathbf{1 8}(200 \mathrm{mg}, 91 \%)$ as a colorless syrup.

$\mathrm{R}_{\mathrm{f}}\left(\mathrm{CH}_{2} \mathrm{Cl}_{2}\right): 0.33$.

Anal. Calcd for $\mathrm{C}_{8} \mathrm{H}_{12} \mathrm{O}_{3}: 70.58 \% \mathrm{C}, 5.88 \%$ H. Found: $707.32 \% \mathrm{C}, 5.74 \% \mathrm{H}$ $\mathrm{RMN}{ }^{1} \mathrm{H}\left(\mathrm{CDCl}_{3}, 400 \mathrm{MHz}\right) \delta$ in ppm: $6.52\left(1 \mathrm{H}, \mathrm{d}, \mathrm{J}_{1,2}=9 \mathrm{~Hz}, \mathrm{H}-1\right), 5.13(1 \mathrm{H}, \mathrm{m}, \mathrm{H}-2)$, 4.51 (1H, m, H-3), 4.20 (1H, m, H-4), 4.05 (1H, m, H-5a), 3.65 (1H, m, H-5b), 1.51, $1.39,\left(6 \mathrm{H}, \mathrm{s}, 2 \mathrm{CH}_{3}\right)$.

$\mathrm{RMN}{ }^{13} \mathrm{C}\left(\mathrm{CDCl}_{3}, 100.6 \mathrm{MHz}\right) \delta$ in ppm: $146.9(\mathrm{C}-1), 108.9\left(\mathrm{C}_{\text {ketal }}\right), 99.2(\mathrm{C}-2), 69.6$ (C-3), $66.8(\mathrm{C}-4), 64.3(\mathrm{C}-5), 27.9,25.5\left(2 \mathrm{CH}_{3}\right)$.

4,6-di-O-benzyl-3-deoxy-D-allal (4,6-di-O-benzyl-D-erythro-pent-1-enitol) (19). ${ }^{5}$

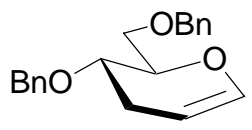

The title compound was prepared following the general procedure above starting from an equimolar mixture of $\mathbf{1 2}(130 \mathrm{mg}, 0.24 \mathrm{mmol})$ in a mixture of $0.5 \mathrm{~mL}$ THF, $20 \mu \mathrm{L}$ acetic acid, NaOAc (27 mg, $0.33 \mathrm{mmol})$ and $160 \mathrm{mg} \mathrm{Zn/Cu}$ couple. The reaction was monitored by TLC (EtOAc/hexane 1:3) and was allowed to reach $15^{\circ} \mathrm{C}$ over a period of $4 \mathrm{~h}$. The crude product was purified by radial chromatography (EtOAc/hexane 1:4) to afford the expected product $19(50 \mathrm{mg}, 71 \%)$ as a colorless syrup.

$\mathrm{R}_{\mathrm{f}}($ EtOAc/hexane 1:3): 0.40 .

Anal. Calcd for $\mathrm{C}_{20} \mathrm{H}_{22} \mathrm{O}_{3}: 77.42 \% \mathrm{C}, 7.10 \% \mathrm{H}$. Found: $77.20 \% \mathrm{C}, 7.01 \% \mathrm{H}$ 
$\mathrm{RMN}^{1} \mathrm{H}\left(\mathrm{CDCl}_{3}, 400 \mathrm{MHz}\right) \delta$ in ppm: 7.35-7.23 $(15 \mathrm{H}, \mathrm{m}, \mathrm{Ar}), 6.61\left(1 \mathrm{H}, \mathrm{d}, \mathrm{J}_{1,2}=6.0\right.$ Hz, H-1), 4.95 (1H, ddd, $\mathrm{J}_{1,2}=6.0$ Hz., $\left.\mathrm{J}_{2,3}=5.2 \mathrm{~Hz} . \mathrm{J}_{2,4}=1.8 \mathrm{~Hz} ., \mathrm{H}-2\right), 4.59-4.27$ (6H, $\left.\mathrm{m}, \mathrm{J}_{\mathrm{AB}}=12.0 \mathrm{~Hz} ., \mathrm{J}_{\mathrm{AB}}{ }^{\prime}=8.6 \mathrm{~Hz} . \mathrm{J}_{\mathrm{AB}}{ }^{\prime}=12.6 \mathrm{~Hz} ., 6 \mathrm{CH}_{2} \mathrm{Ph}\right), 4.16\left(1 \mathrm{H}, \mathrm{ddd}, \mathrm{J}_{4,5}=1.6 \mathrm{~Hz}\right.$, $\left.\mathrm{J}_{5,6 \mathrm{a}}=6.4 \mathrm{~Hz} ., \mathrm{J}_{5,6 \mathrm{~b}}=5.6 \mathrm{~Hz} ., \mathrm{H}-5\right), 3.76\left(1 \mathrm{H}, \mathrm{dd}, \mathrm{J}_{6 \mathrm{a}, 5}=6.4 \mathrm{~Hz} ., \mathrm{J}_{6 \mathrm{a}, \mathrm{b}}=10.0 \mathrm{~Hz} ., \mathrm{H}-6 \mathrm{a}\right)$, $3.74\left(1 \mathrm{H}, \mathrm{d}, \mathrm{J}_{2,3}=5.2 \mathrm{~Hz}, \mathrm{H}-3\right), 3.66\left(1 \mathrm{H}, \mathrm{d}, \mathrm{J}_{4,5}=1.6 \mathrm{~Hz}, \mathrm{H}-4\right), 3.58\left(1 \mathrm{H}, \mathrm{dd}, \mathrm{J}_{5,6 \mathrm{~b}}=5.6\right.$ Hz., $\left.\mathrm{J}_{6 \mathrm{a}, \mathrm{b}}=10.0 \mathrm{~Hz} ., \mathrm{H}-6 \mathrm{~b}\right)$.

$\mathrm{RMN}{ }^{13} \mathrm{C}\left(\mathrm{CDCl}_{3}, 100.6 \mathrm{MHz}\right) \delta$ in ppm: $146.9(\mathrm{C}-1), 138.5-127.9\left(\mathrm{C}_{\text {aromatic }}\right), 98.7$ (C2), $73.6\left(\mathrm{CH}_{2} \mathrm{Ph}\right), 73.0$ (C-5), 72.7 (C-4), 72.4, $70.0\left(2 \mathrm{CH}_{2} \mathrm{Ph}\right), 69.1$ (C-6), $67.3(\mathrm{C}-3)$.

Phenyl 4,6-di-O-benzyl-2,3-dideoxy-1-thio- $\alpha / \beta$-D-ribo-pyranoside (22).

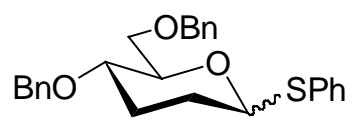

The title compound was prepared following the general procedure above starting from an equimolar mixture of 21 (40 mg, $0.08 \mathrm{mmol}$ ) in a mixture of $0.5 \mathrm{~mL} \mathrm{THF,} 7 \mu \mathrm{L}$ acetic acid, $\mathrm{NaOAc}(9.6 \mathrm{mg}, 0.12 \mathrm{mmol})$ and $53 \mathrm{mg} \mathrm{Zn} / \mathrm{Cu}$ couple for $1.5 \mathrm{~h}$. The reaction was monitored by TLC (EtOAc/hexane 1:4). The crude product was purified by radial chromatography (EtOAc/hexane 1:5) to afford the product $22(31 \mathrm{mg}, 88 \%)$ as a $1: 1 \alpha / \beta$ anomeric mixture as a colorless syrup.

Anal. Calcd for $\mathrm{C}_{26} \mathrm{H}_{26} \mathrm{O}_{3} \mathrm{~S}: 74.26 \%$ C, $6.67 \% \mathrm{H}, 7.62 \%$ S. Found: $74.42 \% \mathrm{C}, 6.75 \% \mathrm{H}$, $7.75 \% \mathrm{~S}$.

RMN ${ }^{1} \mathrm{H}\left(\mathrm{CDCl}_{3}, 400 \mathrm{MHz}\right) \delta$ in ppm: 7.61-7.22 (15H, m, Ar), $4.71\left(1 \mathrm{H}, \mathrm{d}, \mathrm{J}_{1,2 \mathrm{a}}=10.4\right.$ $\mathrm{Hz}, \mathrm{H}-1), 4.61-4.40\left(4 \mathrm{H}, \mathrm{m}, \mathrm{J}_{\mathrm{AB}}=11.6 \mathrm{~Hz} ., \mathrm{J}_{\mathrm{AB}}\right.$ = $\left.12.0 \mathrm{~Hz} ., 4 \mathrm{CH}_{2} \mathrm{Ph}\right), 3.80-3.68(4 \mathrm{H}, \mathrm{m}$, , H-2a, H-2b, H-6a, H-6b), 3.58 (1H, ddd, $\mathrm{J}_{5,4}=9.6$ Hz., $\mathrm{J}_{5,6 \mathrm{a}}=2.0 \mathrm{~Hz} ., \mathrm{J}_{5,6 \mathrm{~b}}=4.8 \mathrm{~Hz} ., \mathrm{H}-$ 5), 3.47 (1H, ddd, $\left.\mathrm{J}_{4,5}=9.6 \mathrm{~Hz} ., \mathrm{J}_{4,3 \mathrm{a}}=4.4 \mathrm{~Hz} ., \mathrm{J}_{4,3 \mathrm{~b}}=10.4 \mathrm{~Hz} ., \mathrm{H}-4\right), 2.88$ (1H, ddd, $\left.\mathbf{J}_{3 \mathrm{a}, 3 \mathrm{~b}}=12.4 \mathrm{~Hz} ., \mathbf{J}_{3 \mathrm{a}, 2 \mathrm{a}}=4.8 \mathrm{~Hz} ., \mathbf{J}_{3 \mathrm{a}, 4}=4.4 \mathrm{~Hz} ., \mathrm{H}-3 \mathrm{a}\right), 2.03\left(1 \mathrm{H}\right.$, ddd, $\mathbf{J}_{3 \mathrm{~b}, 3 \mathrm{a}}=12.4 \mathrm{~Hz}$, $\left.\mathrm{J}_{3 \mathrm{~b}, 4}=10.4 \mathrm{~Hz} ., \mathrm{J}_{3 \mathrm{~b}, 2 \mathrm{a}}=11.2 \mathrm{~Hz} ., \mathrm{H}-3 \mathrm{~b}\right)$.

$\mathrm{RMN}{ }^{13} \mathrm{C}\left(\mathrm{CDCl}_{3}, 100.6 \mathrm{MHz}\right) \delta$ in ppm: 133.4-127.8 ( $\left.\mathrm{C}_{\text {aromatic }}\right), 89.5$ (C-1), 82.0 (C-5), 73.6 (C-4), 72.7, 71.7 (2 $\left.\mathrm{CH}_{2} \mathrm{Ph}\right), 69.3$ (C-6), 45.7 (C-2), 41.9 (C-3). 


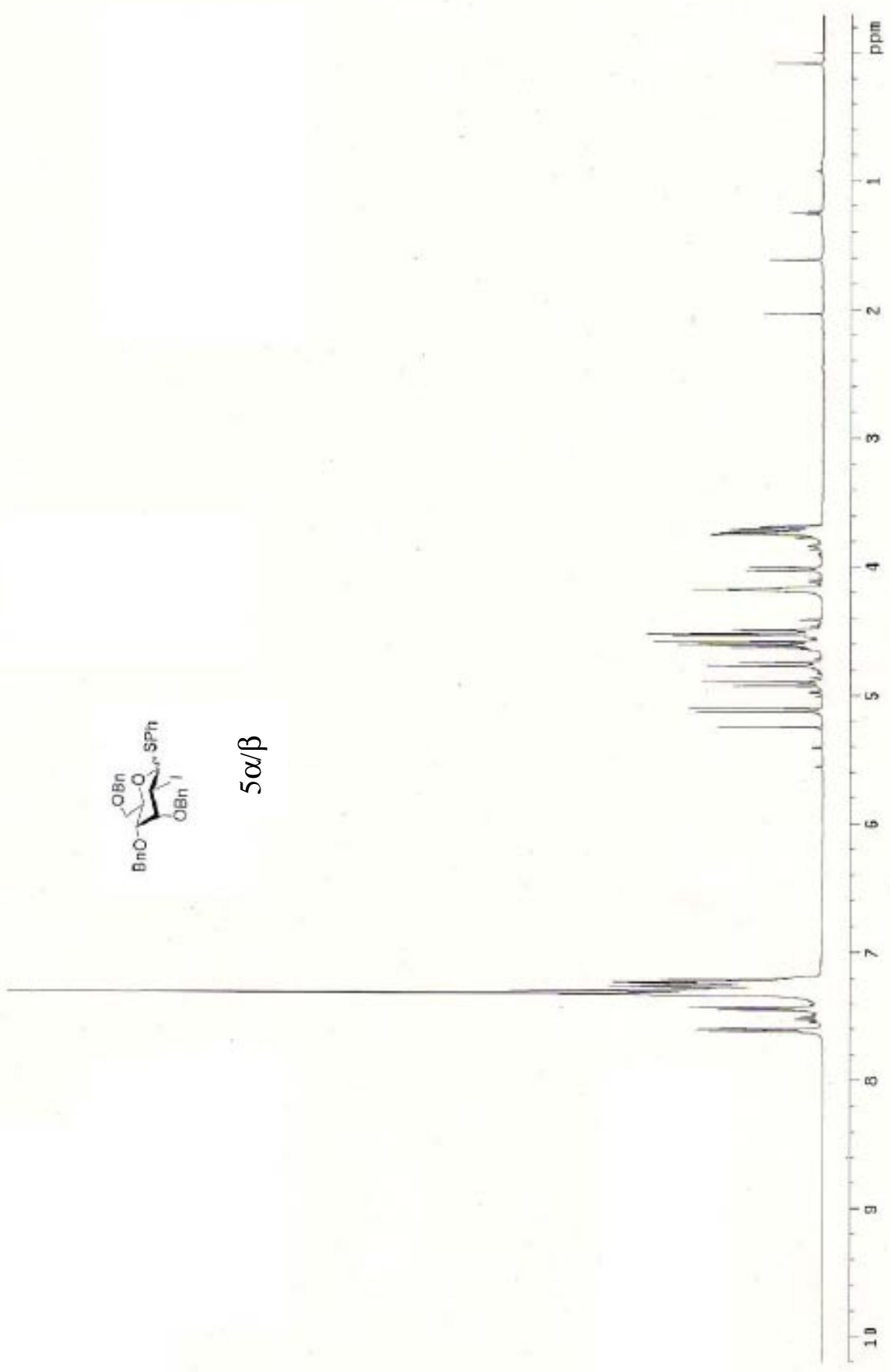




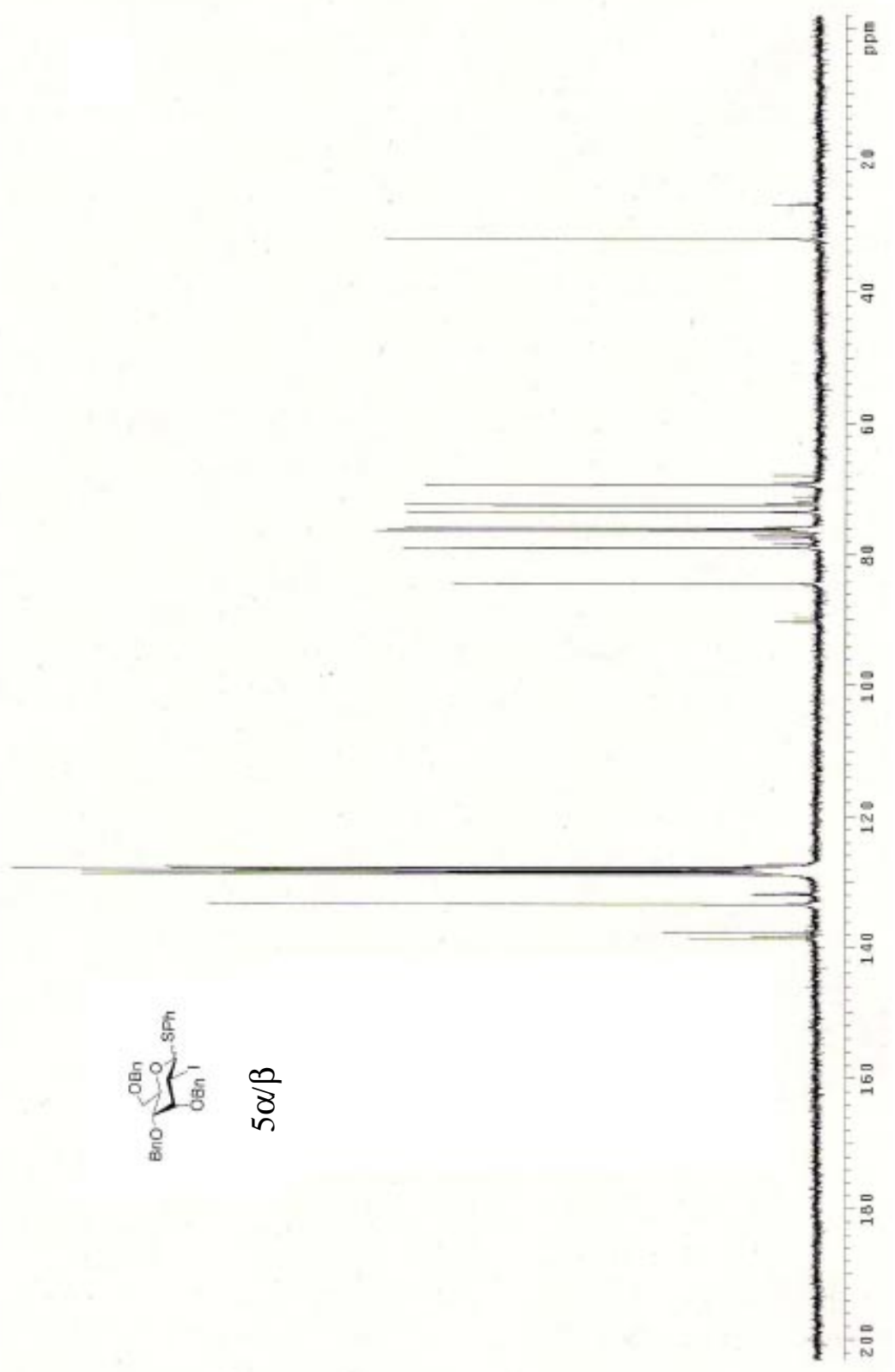

is 


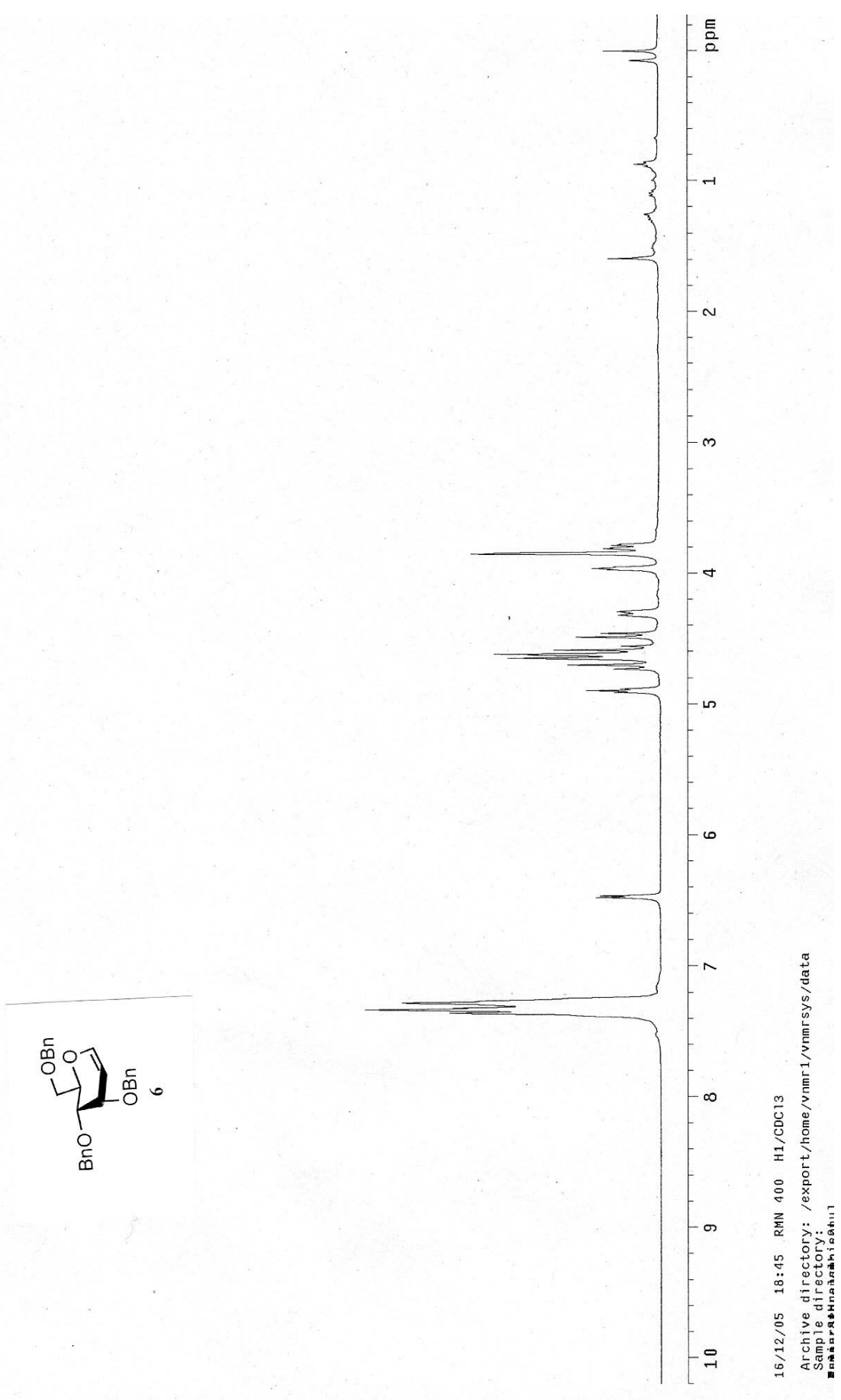

$\frac{0}{n}$ 


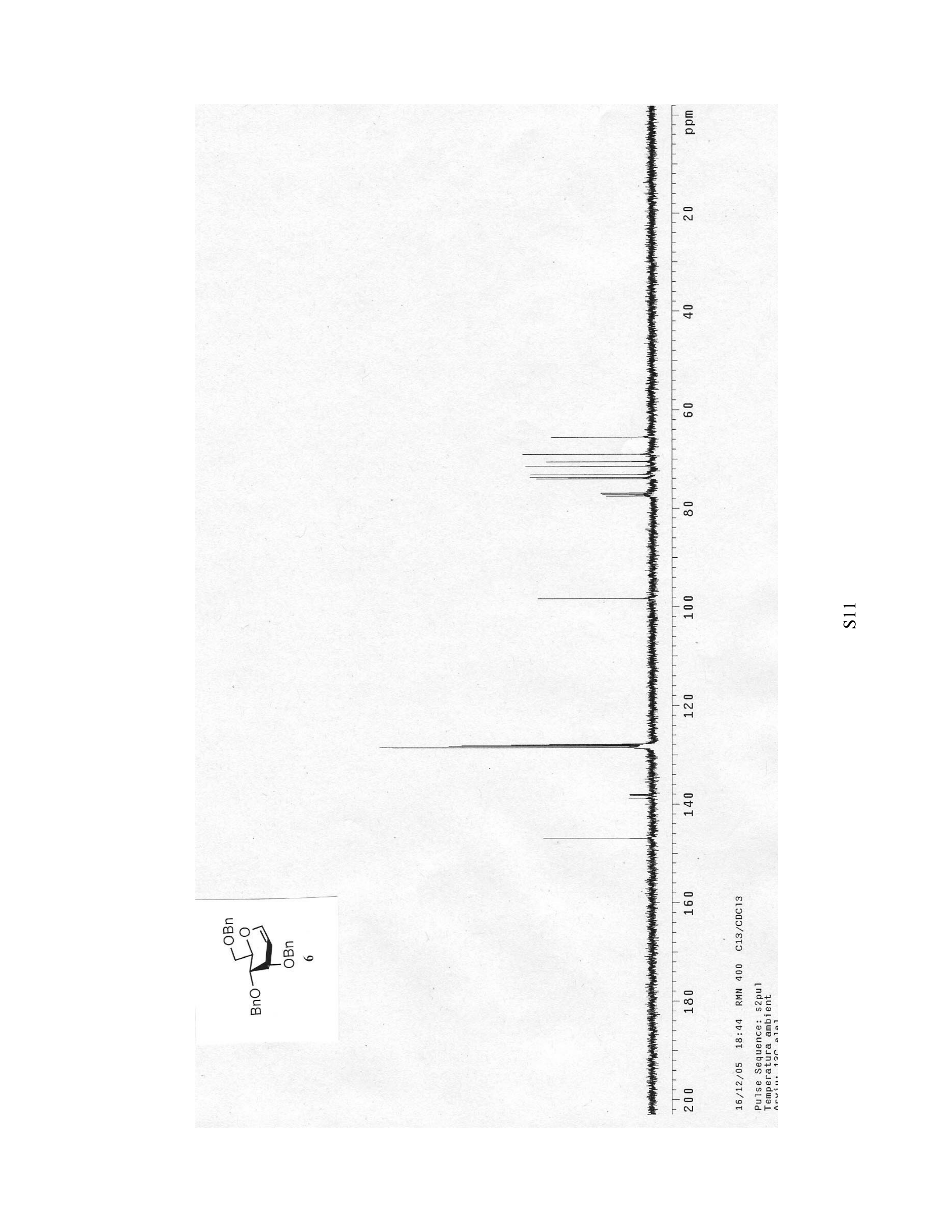




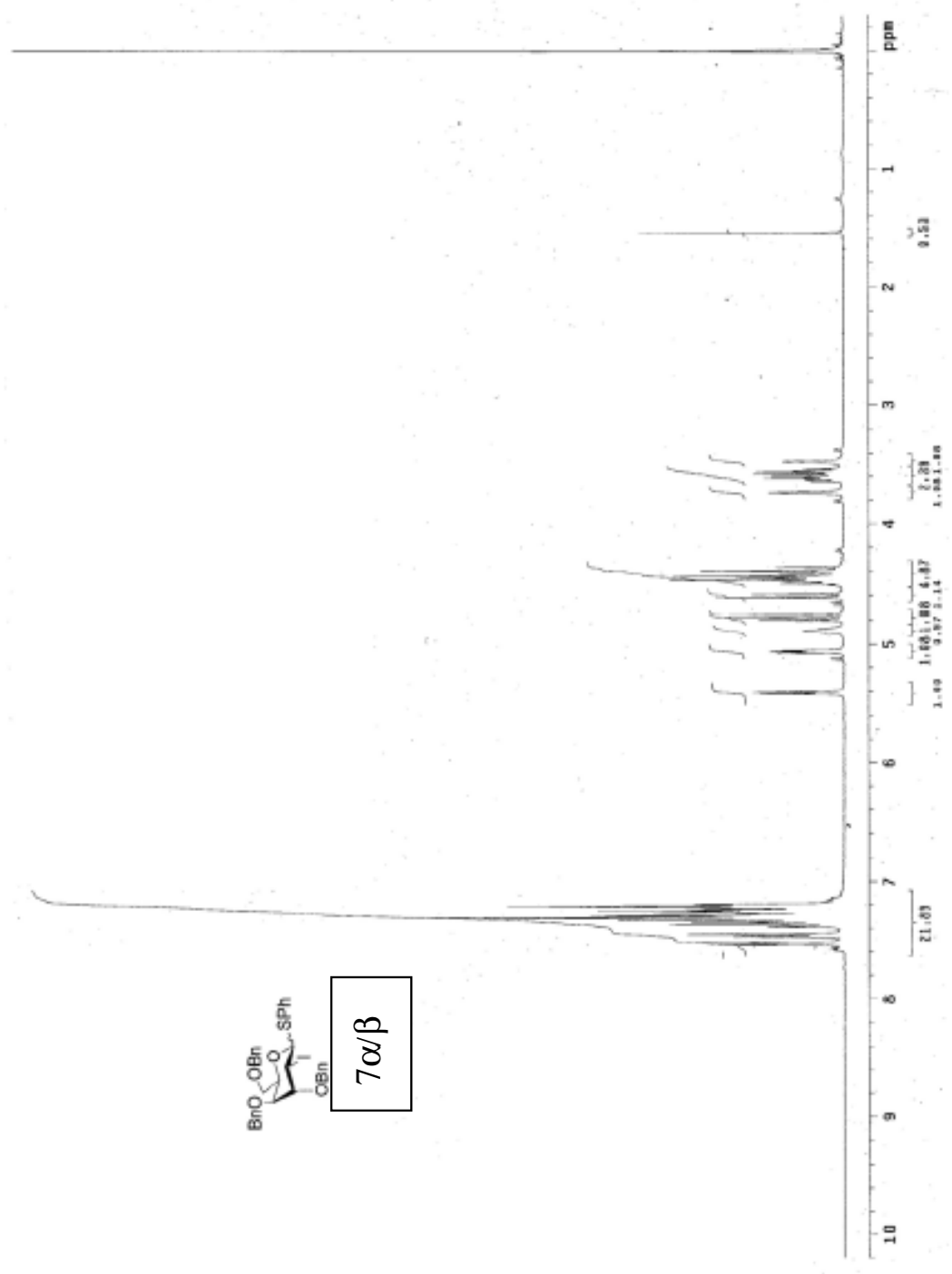

क 


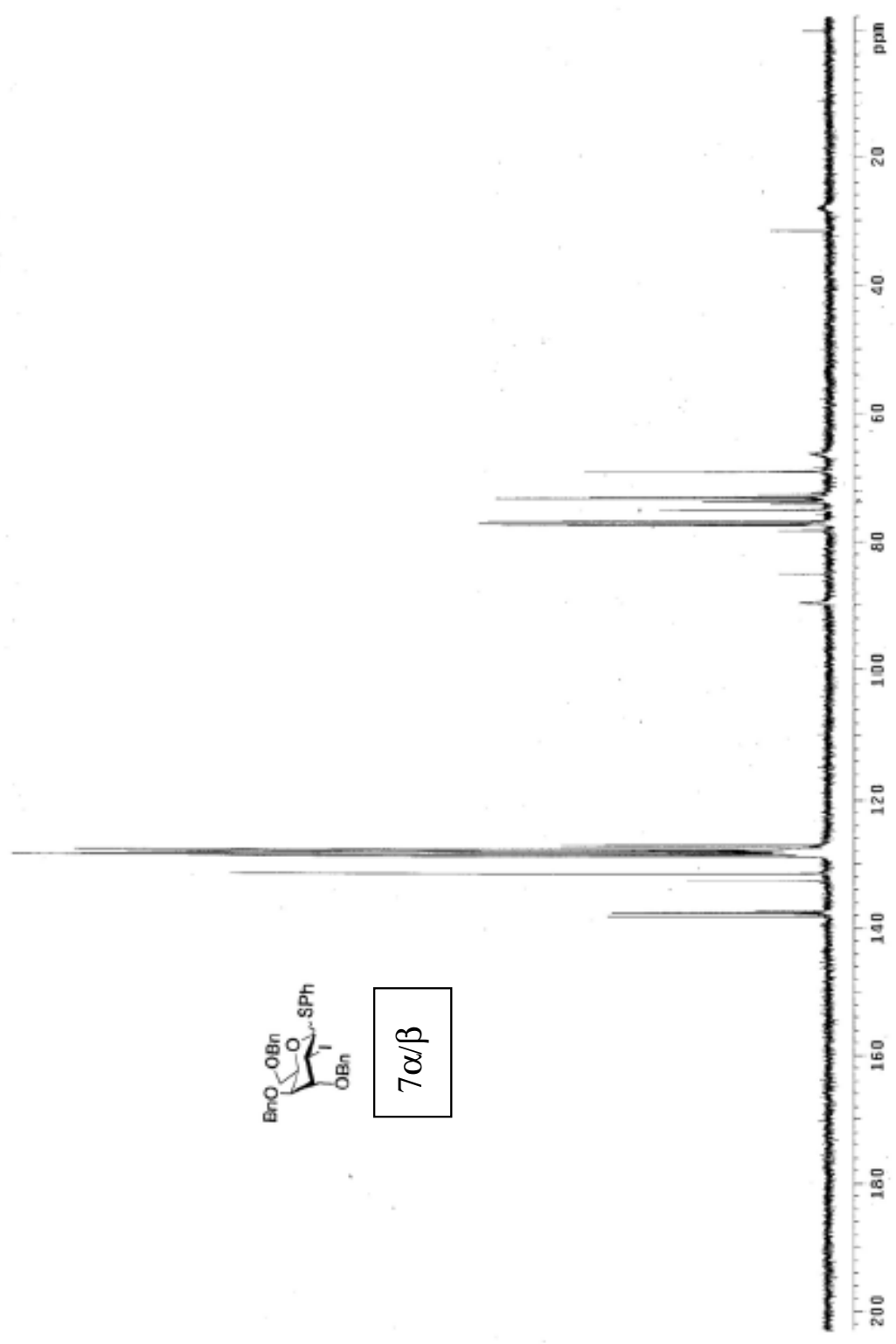




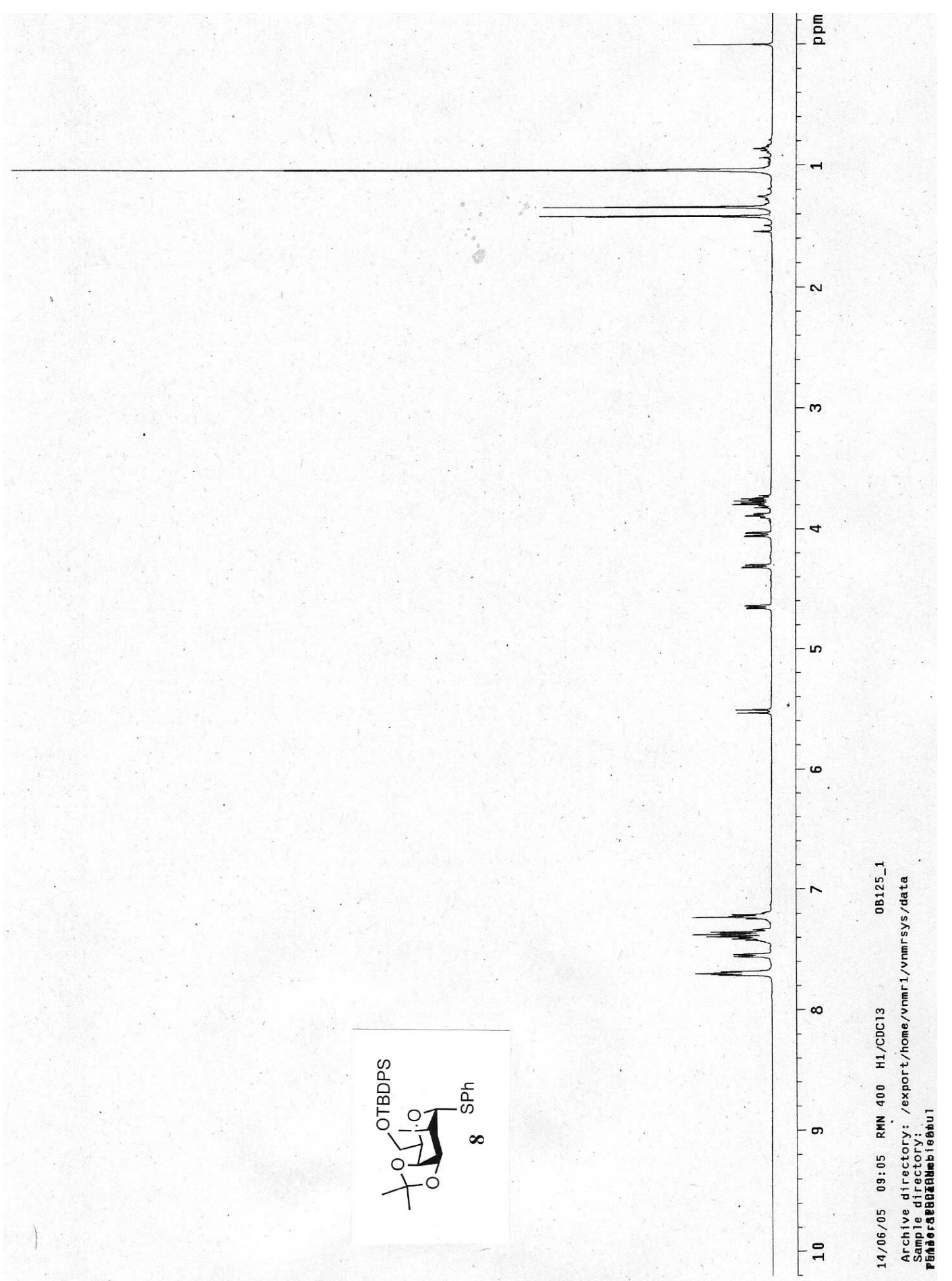




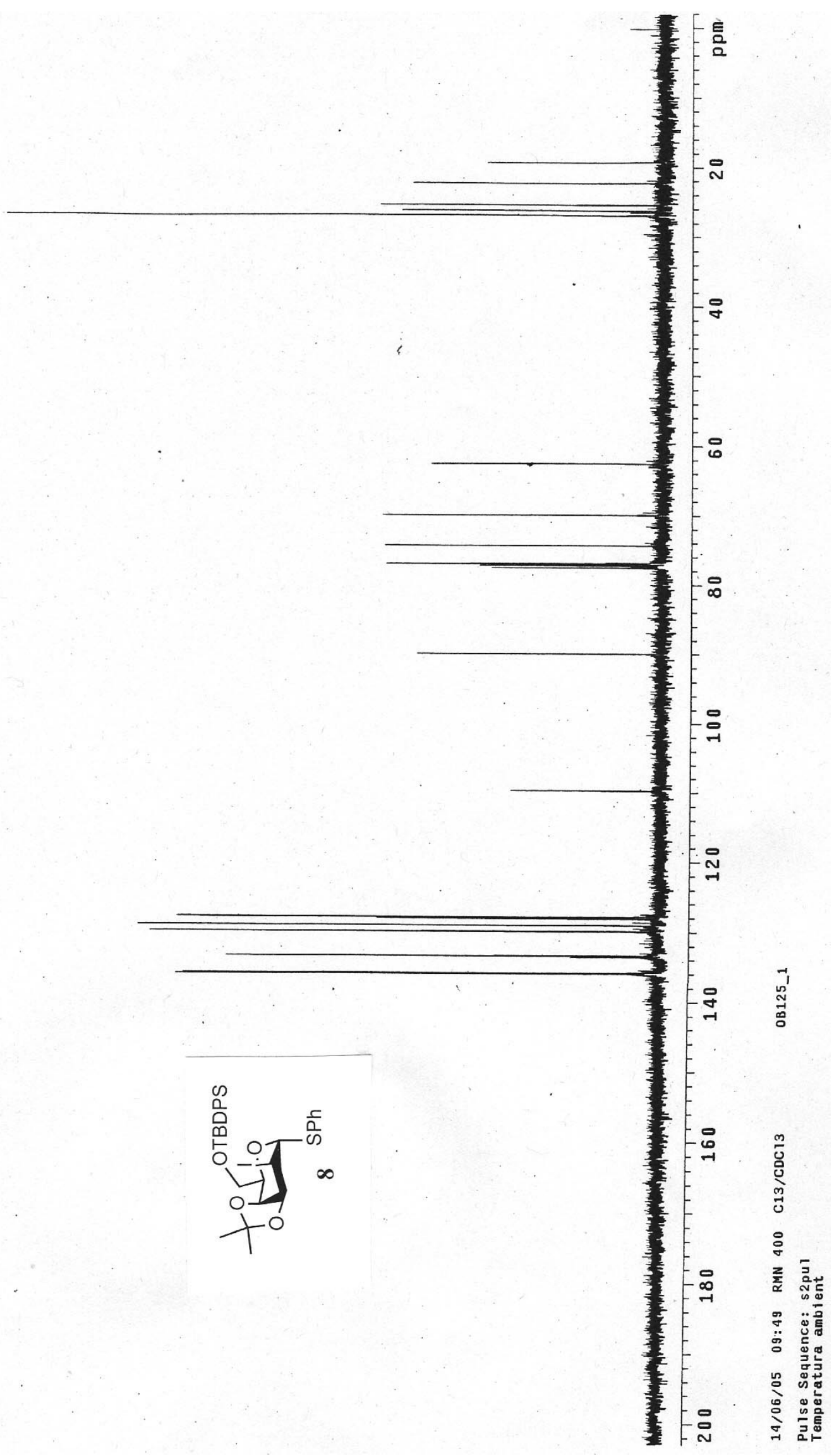




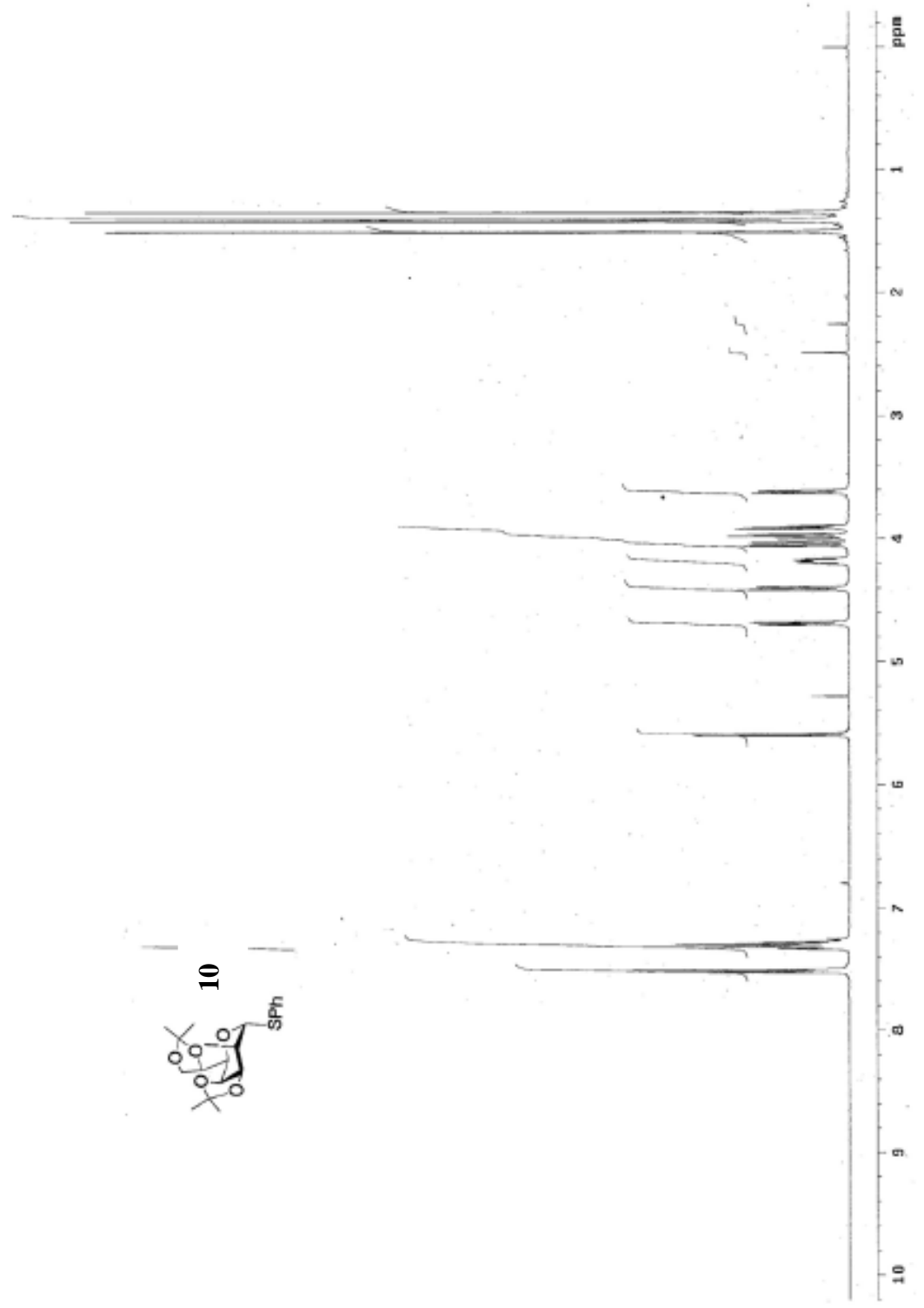



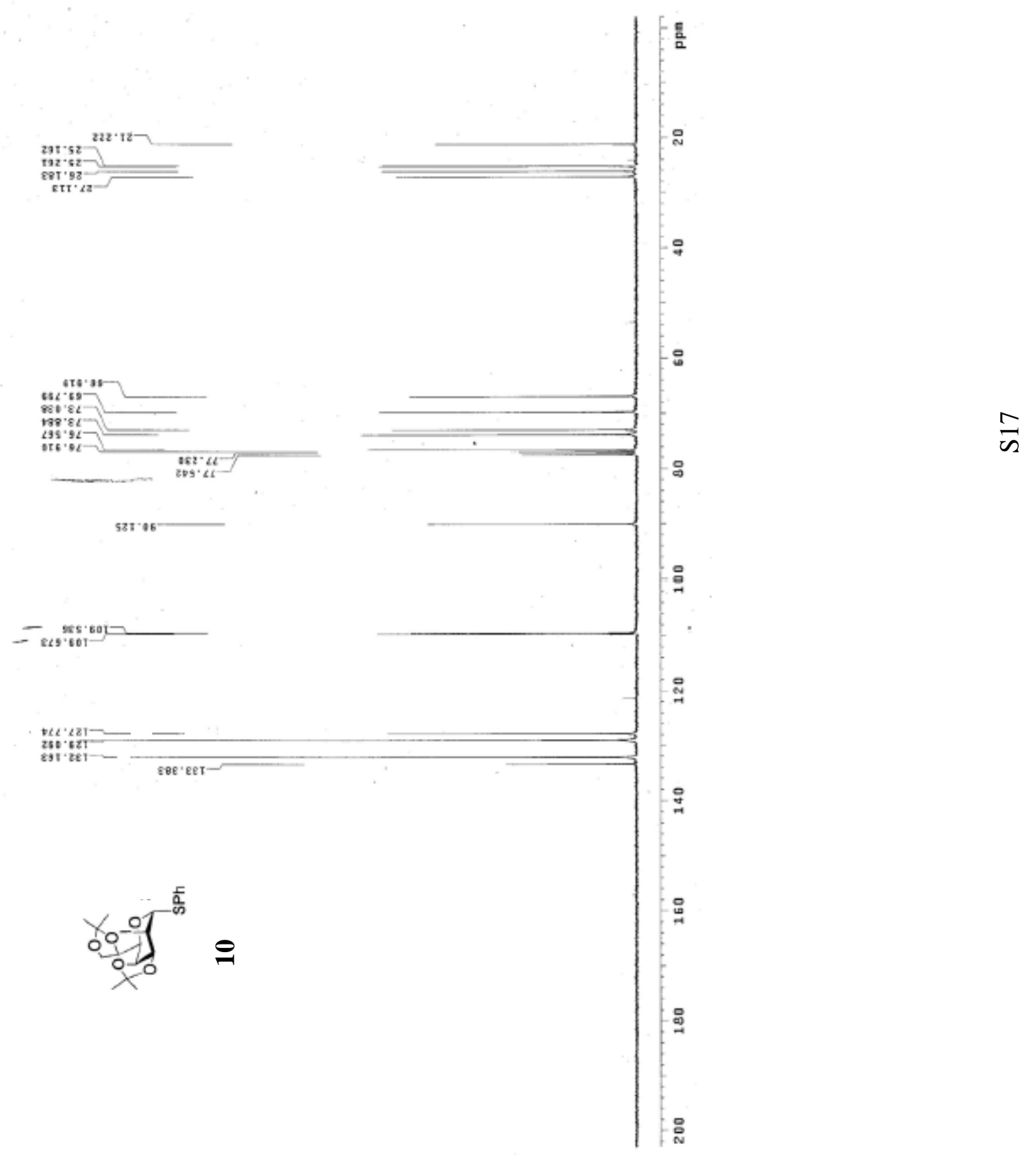


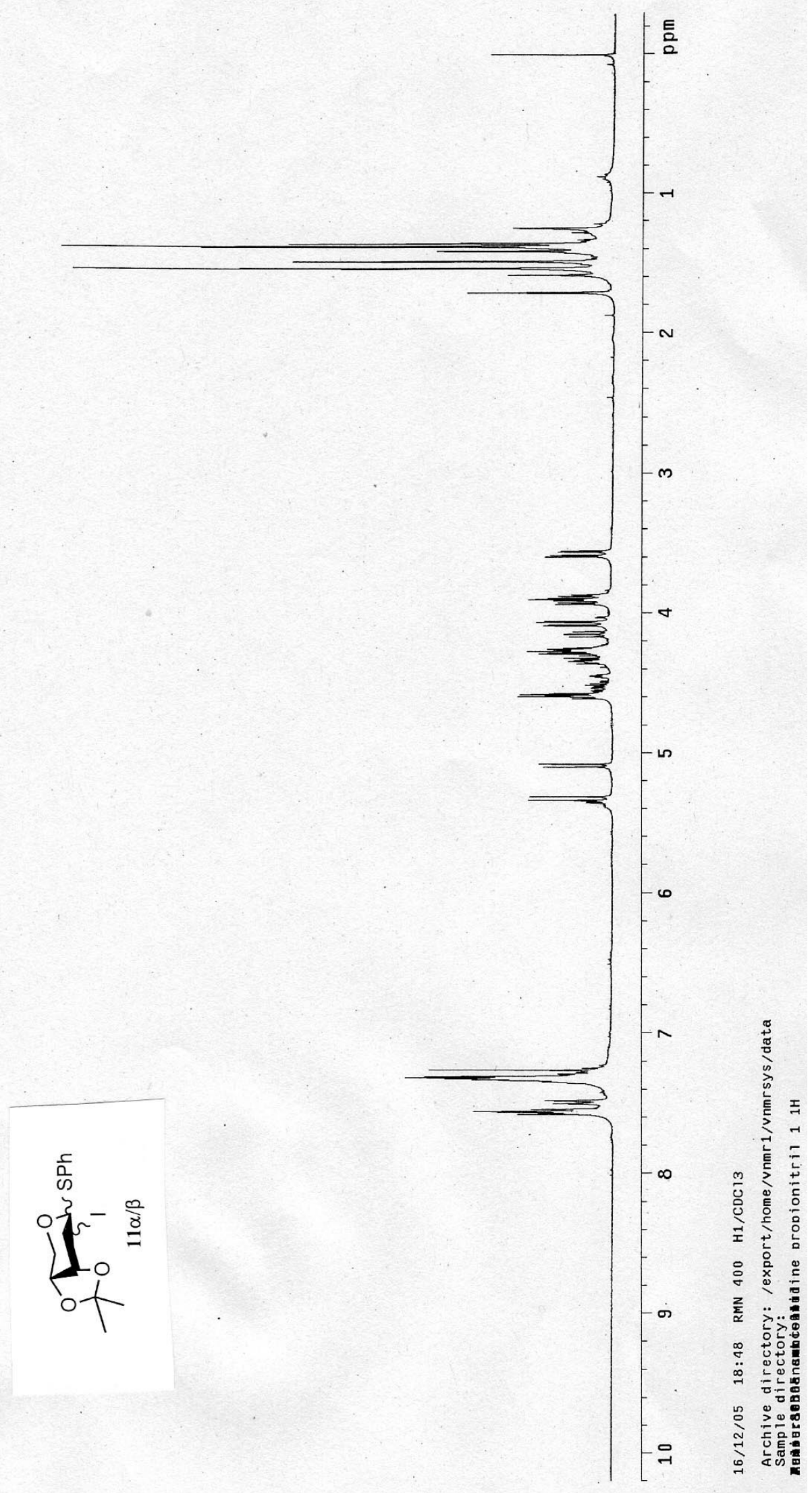




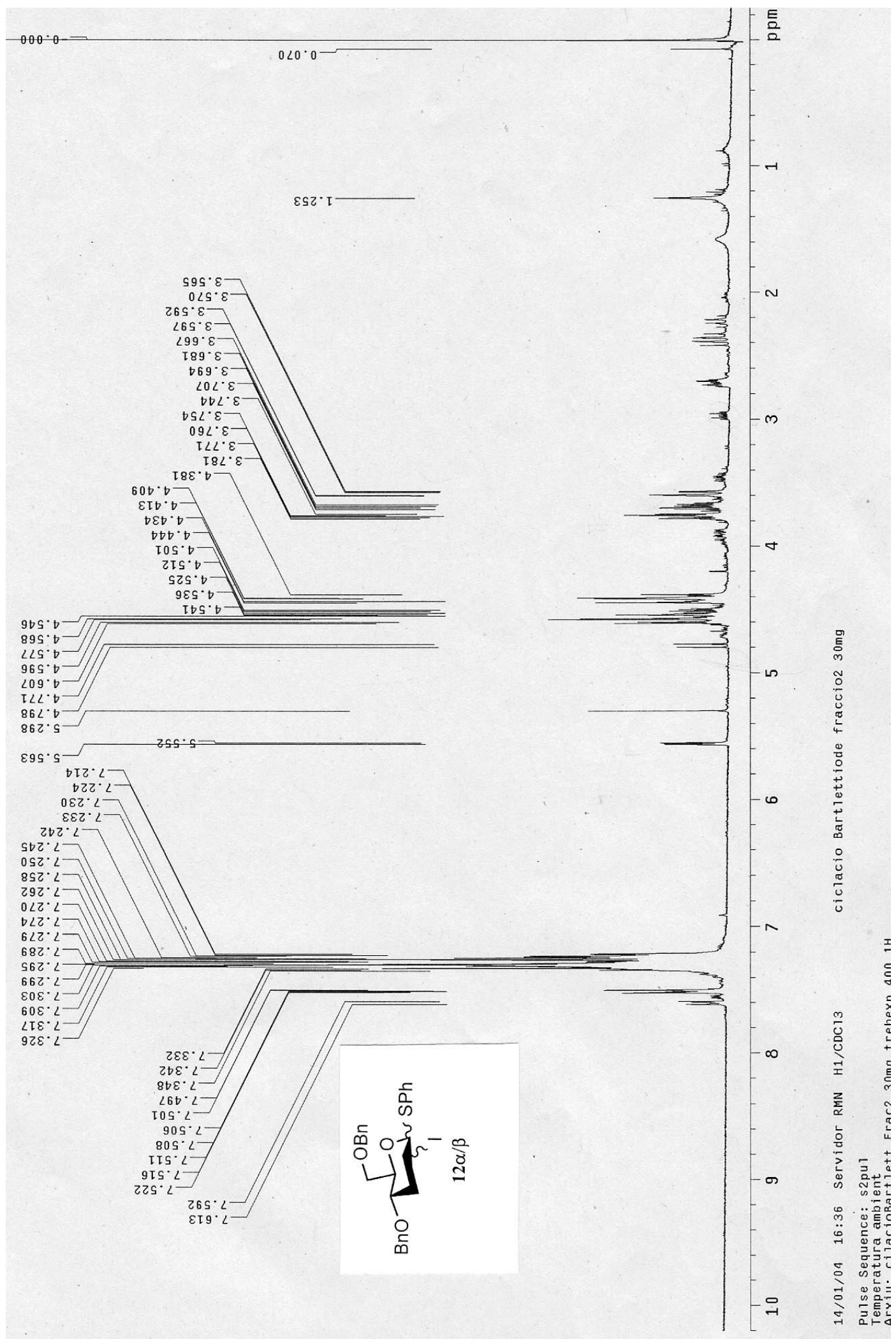




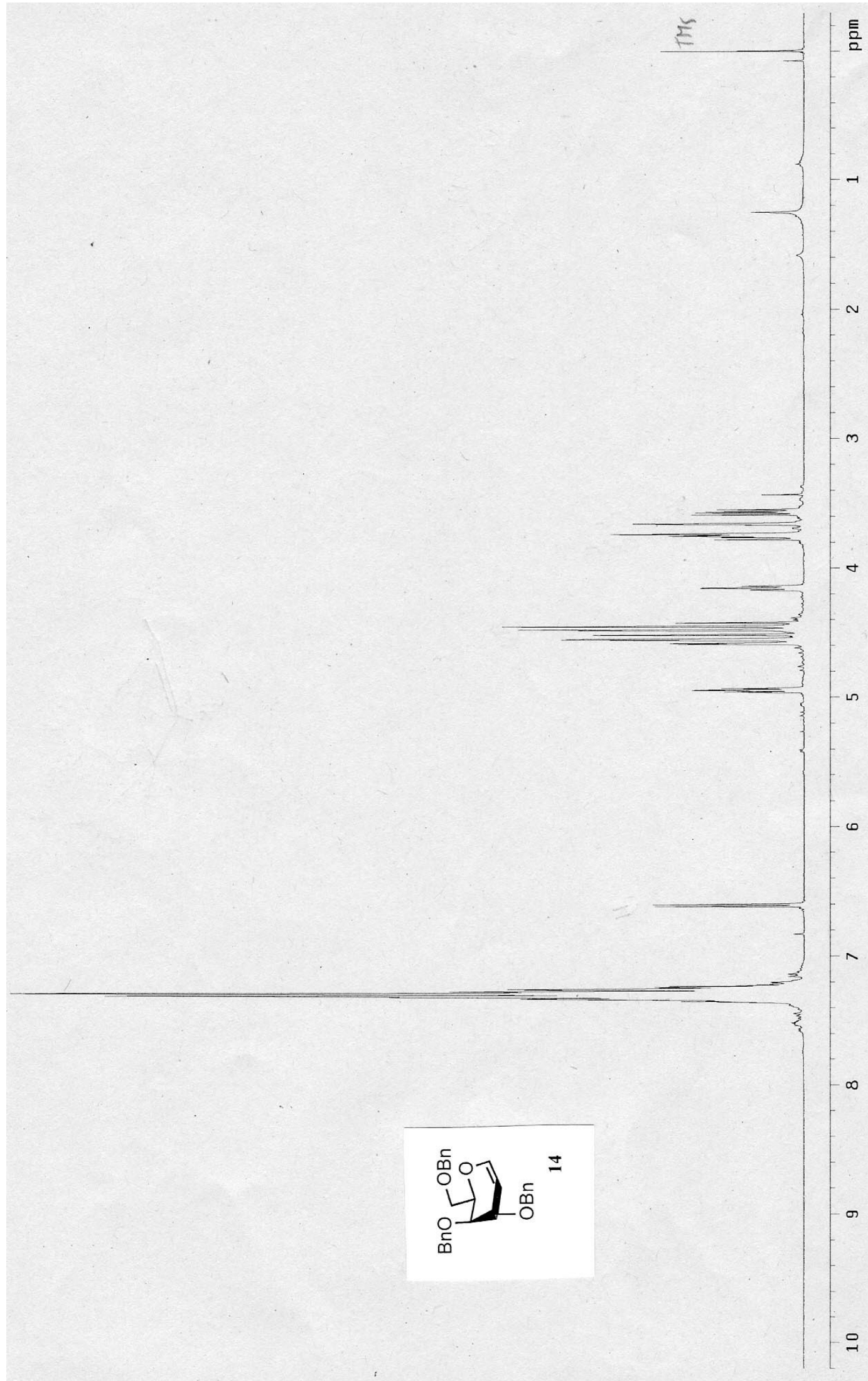

\&: 


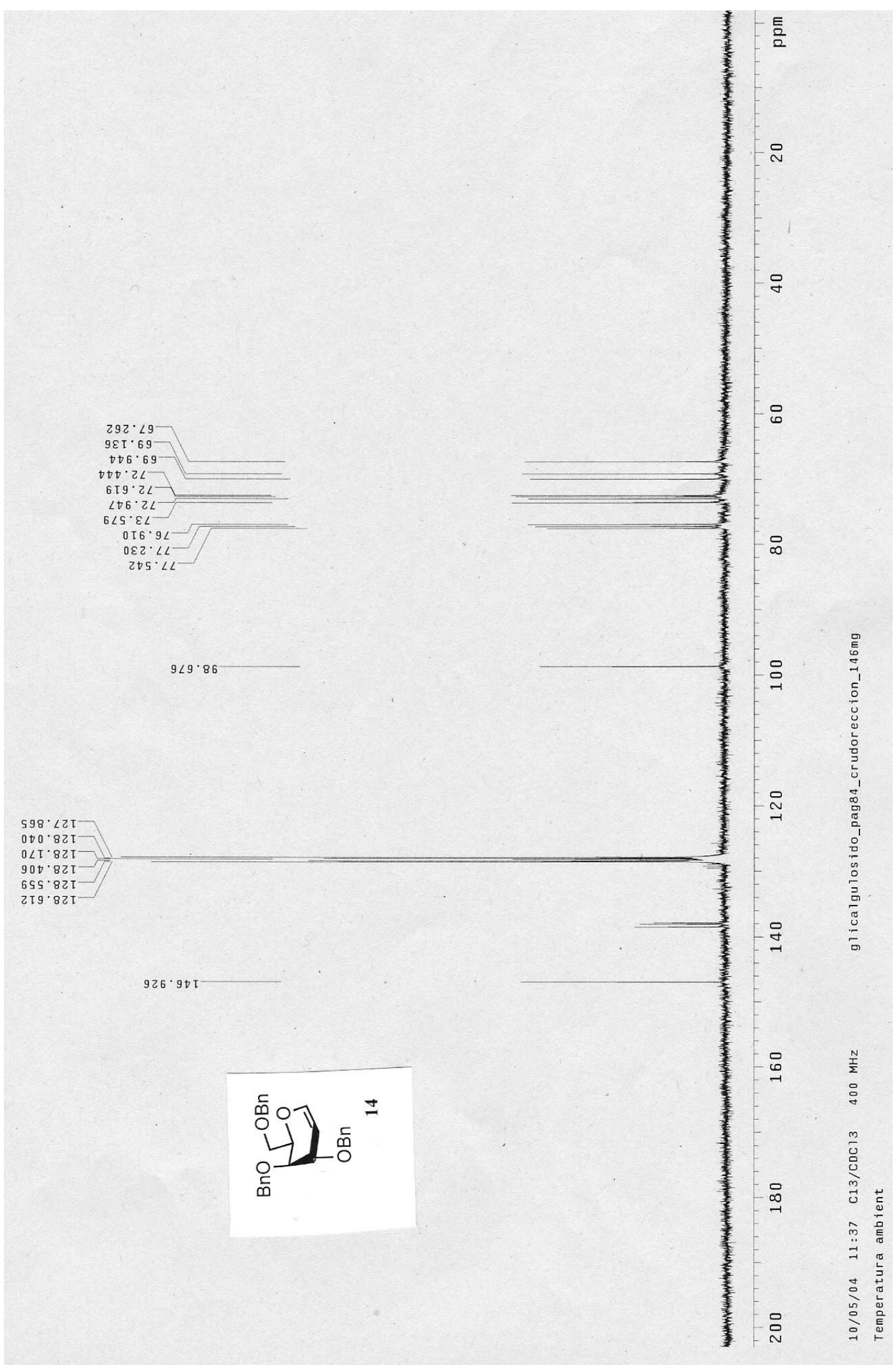




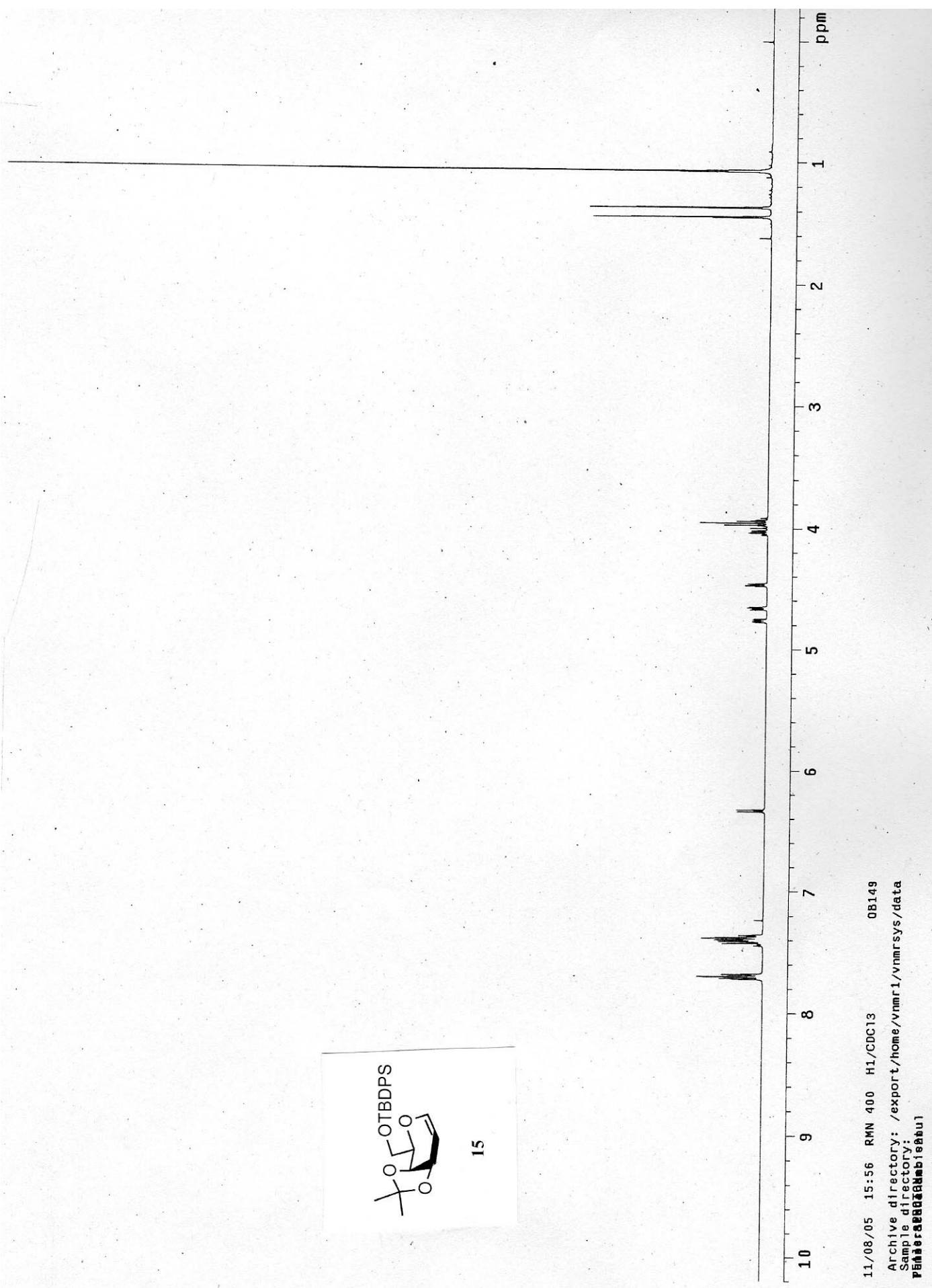




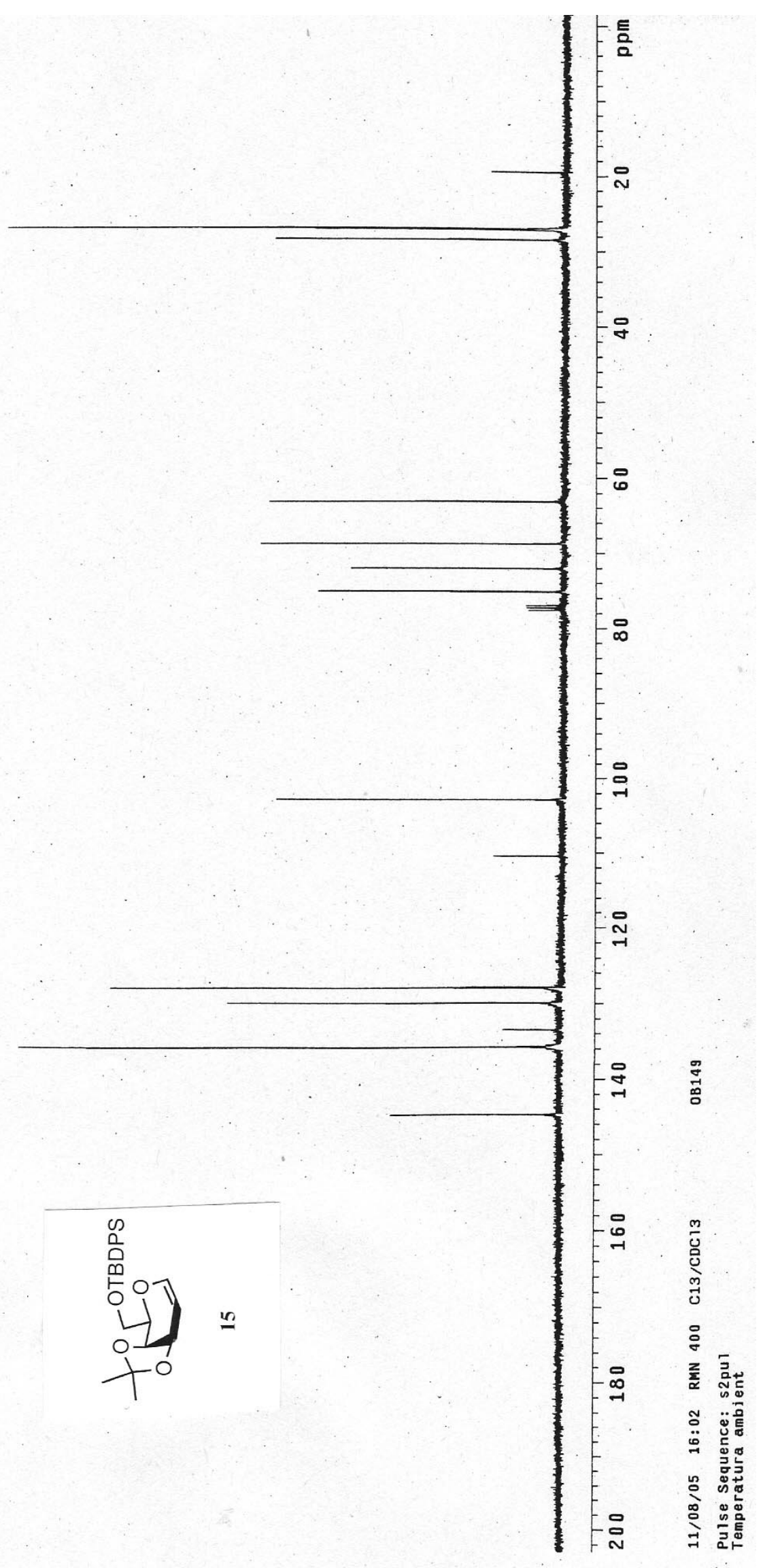




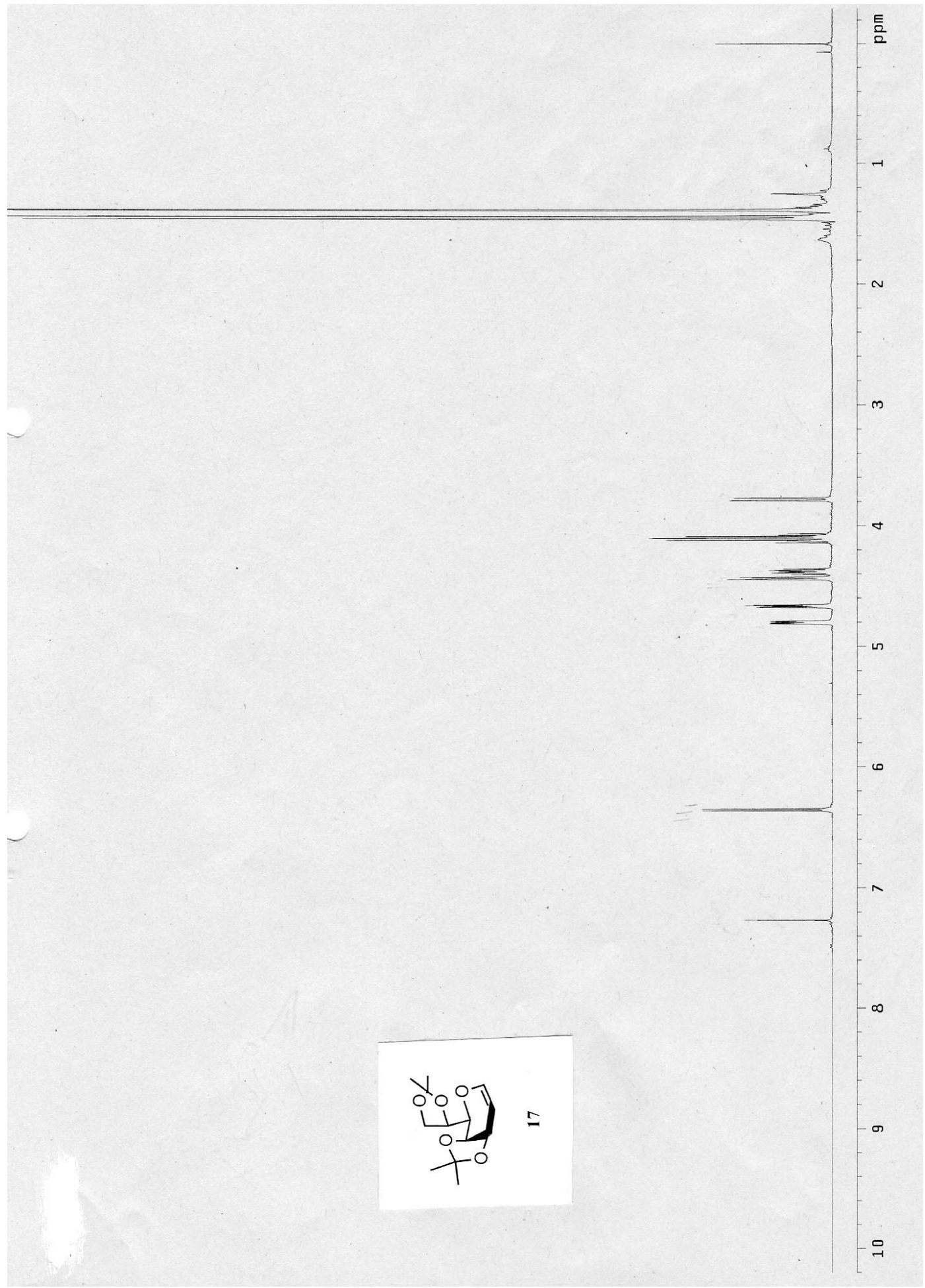




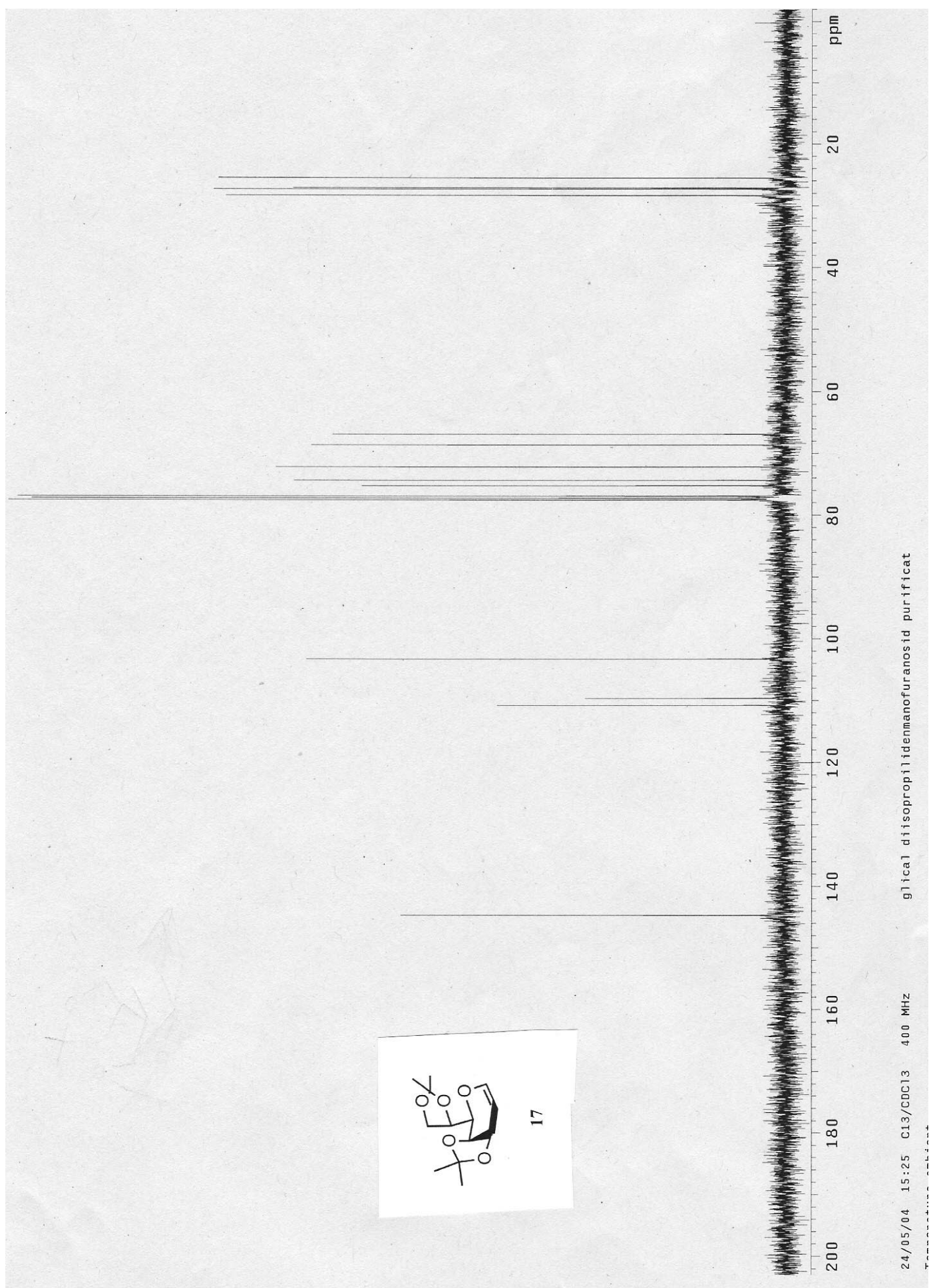




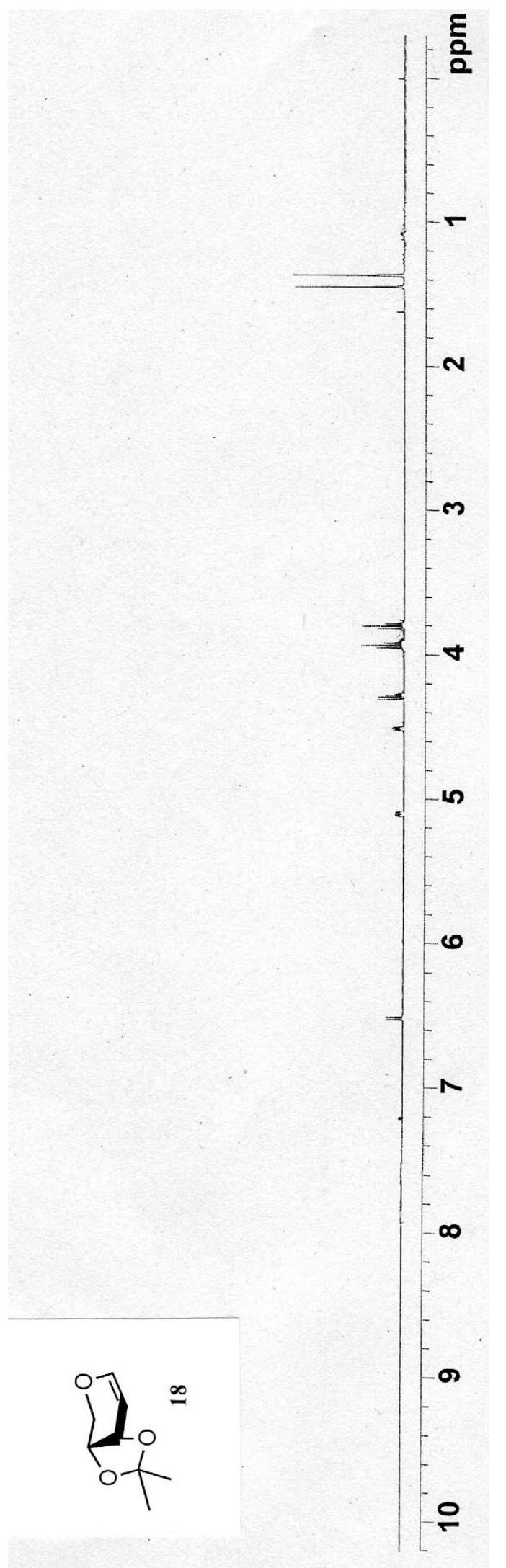




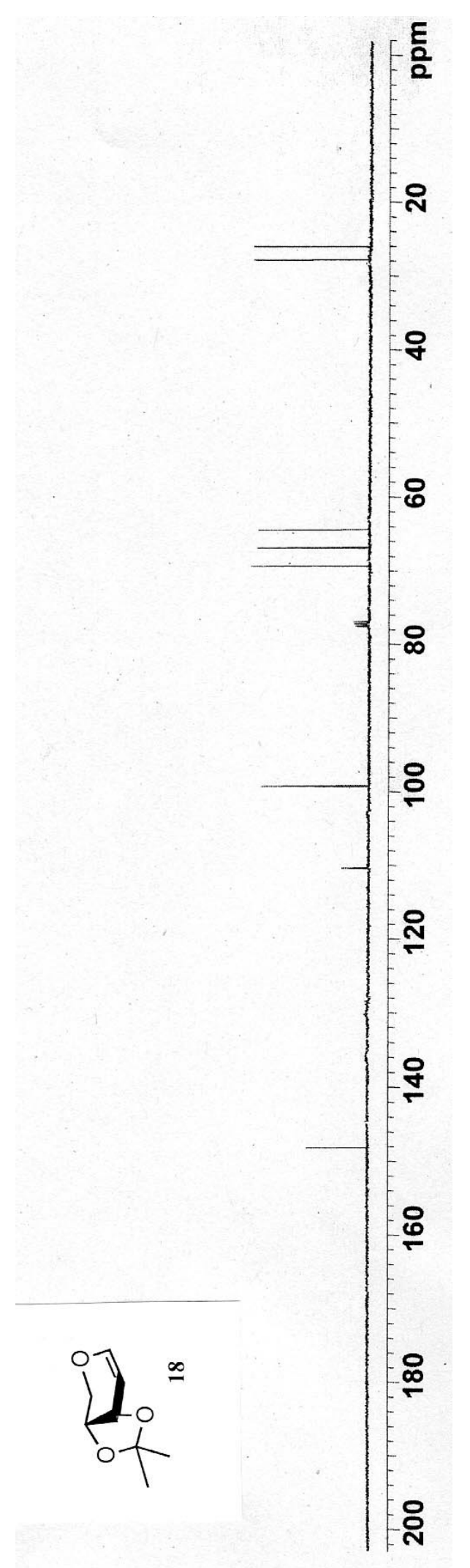




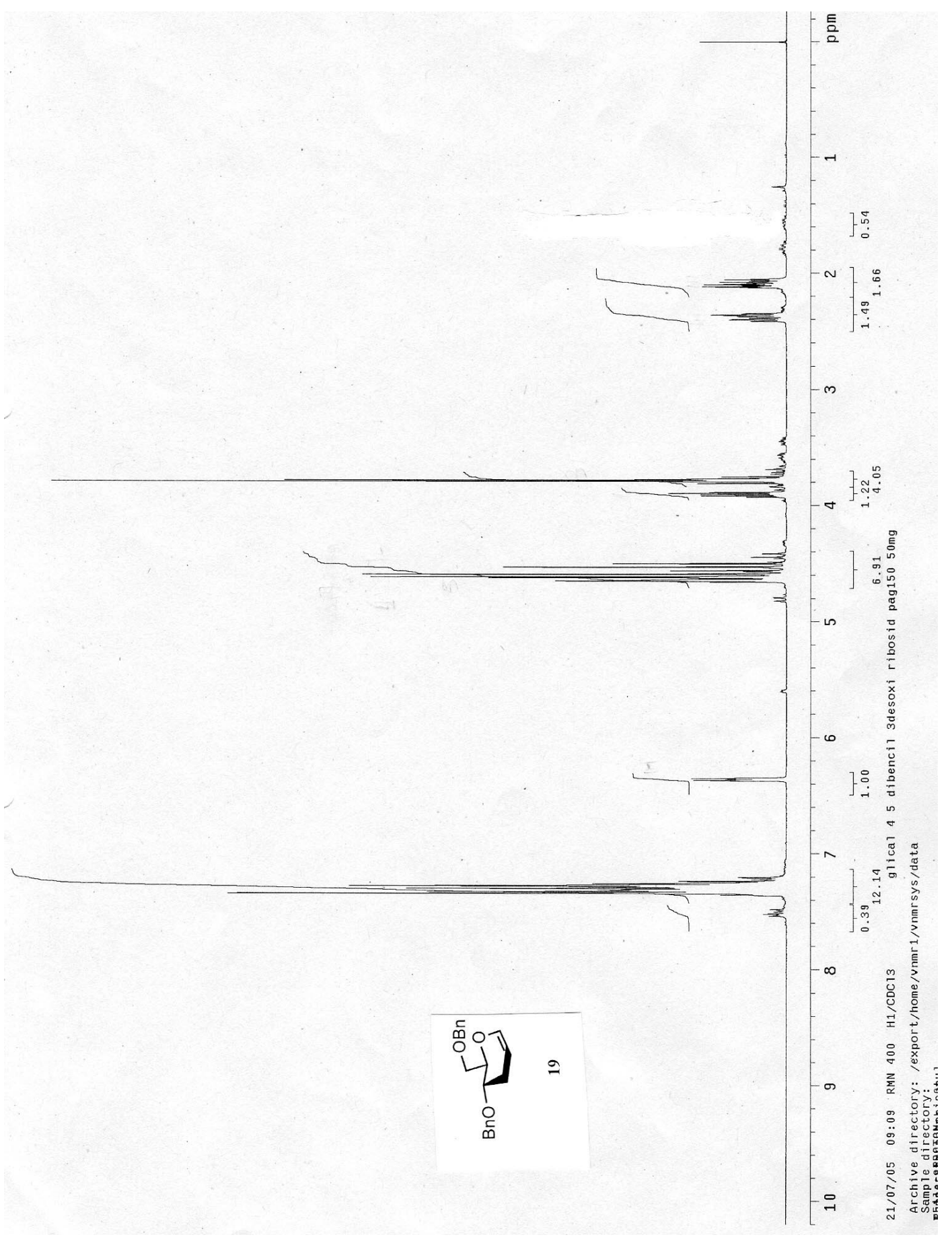




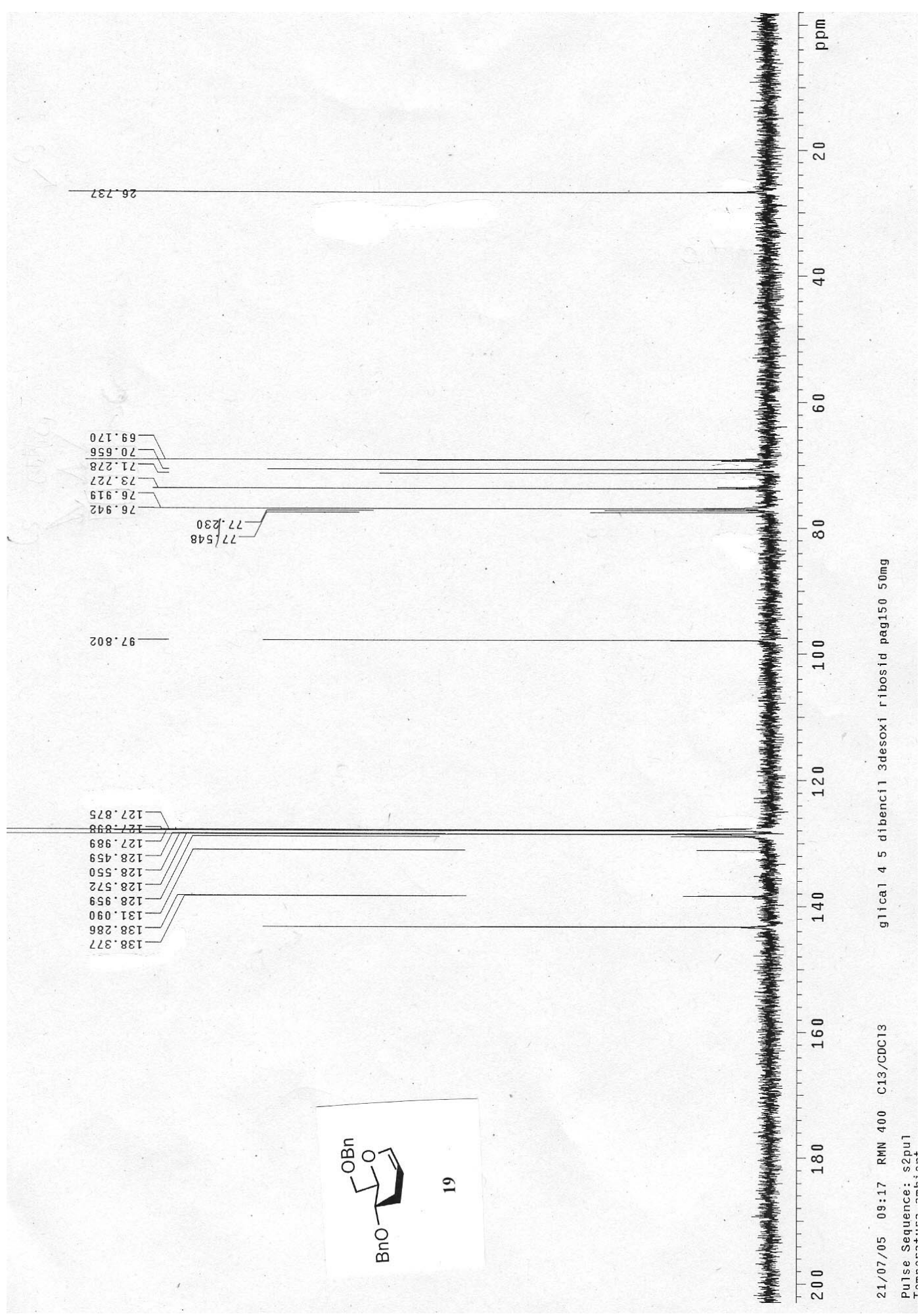

๙ิ 


\section{References}

${ }^{1}$ a) Tamarez, M.M.;Franck, R.W.; Geer, A. Tetrahedron 2003, 59, 4249-4259. b) Dios, A.; Franck, R.W.; Capossi, G.; Menichetti, S.; Nativi, C. Org. Lett. 1999, 1, 111-113. C. Grenwal, G.; Franck, R.W. J. Org. Chem. 1992, 57, 2084.

${ }^{2}$ Broddefalk, J.; Bergquist, K-E.; Kihlberg, J. Tetrahedron, 1998, 54, 12047-12050.

${ }^{3}$ Wardrop, D.J.; Zhang, W. Tetrahedron Lett. 2002, 43, 5389-5391.

${ }^{4}$ Cook, M.J.; Fletcher, M.J.E.; Gray, D.; Lovell, P.J.; Gallagher, T. Tetrahedron 2004, 60, 5085-5092.

${ }^{5}$ a) Gopal Reddy, B.; Vankar, Y.D. Tetrahedron Lett. 2003. 44, 4765-4767. b) Fraser-Reid, B.; Radatus, B. J. Am. Chem. Soc. 1970, 92, 6661-6663. 